\title{
Constructible functions on Artin stacks
}

\author{
Dominic Joyce \\ Lincoln College, Oxford
}

\section{Introduction}

Let $\mathbb{K}$ be an algebraically closed field, $X$ a $\mathbb{K}$-scheme, and $X(\mathbb{K})$ the set of closed points in $X$. A constructible set $C \subseteq X(\mathbb{K})$ is a finite union of subsets $Y(\mathbb{K})$ for finite type $\mathbb{K}$-subschemes $Y$ in $X$. A constructible function $f: X(\mathbb{K}) \rightarrow \mathbb{Q}$ has $f(X(\mathbb{K}))$ finite and $f^{-1}(c)$ constructible for all $0 \neq c \in f(X(\mathbb{K}))$. Write $\mathrm{CF}(X)$ for the $\mathbb{Q}$-vector space of constructible functions on $X$.

Let $\phi: X \rightarrow Y$ and $\psi: Y \rightarrow Z$ be morphisms of $\mathbb{C}$-varieties. MacPherson [16, Prop. 1] defined a $\mathbb{Q}$-linear pushforward $\mathrm{CF}(\phi): \mathrm{CF}(X) \rightarrow \mathrm{CF}(Y)$ with $\left(\mathrm{CF}(\phi) \delta_{W}\right)(y)=\chi_{\text {an }}\left(\phi^{-1}(y) \cap W\right)$ for subvarieties $W$ in $X$ and $y \in Y(\mathbb{C})$, where $\chi_{\text {an }}$ is the topological Euler characteristic in compactly-supported cohomology with the analytic topology, and $\delta_{W}$ the characteristic function of $W(\mathbb{C})$ in $X(\mathbb{C})$. It satisfies $\mathrm{CF}(\psi \circ \phi)=\mathrm{CF}(\psi) \circ \mathrm{CF}(\phi)$, so that $\mathrm{CF}$ is a functor from the category of $\mathbb{C}$-varieties to the category of $\mathbb{Q}$-vector spaces. This was extended to other fields $\mathbb{K}$ of characteristic zero by Kennedy [13].

This paper generalizes these results to $\mathbb{K}$-schemes and algebraic $\mathbb{K}$-stacks in the sense of Artin, for $\mathbb{K}$ of characteristic zero. We introduce a notion of pseudomorphism $\Phi$ between locally constructible sets in $\mathbb{K}$-schemes or $\mathbb{K}$-stacks, generalizing morphisms. Pushforwards $\mathrm{CF}(\Phi)$ exist, and pseudomorphisms seem very natural for constructible functions problems.

The motivation for this is my series of papers [8-11]. Let $\operatorname{coh}(P)$ be the abelian category of coherent sheaves on a projective $\mathbb{K}$-scheme $P$, and $(\tau, T, \leqslant)$ a stability condition on $\operatorname{coh}(P)$. Then the moduli space $\mathfrak{O} \mathfrak{b j} \mathfrak{j}_{\operatorname{coh}(P)}$ of sheaves in $\operatorname{coh}(P)$ is an Artin $\mathbb{K}$-stack, and the set $\operatorname{Obj}_{\mathrm{ss}}^{\alpha}(\tau)$ of $\tau$-semistable sheaves in class $\alpha$ is a constructible subset in $\mathfrak{O b j}_{\operatorname{coh}(P)}$. We shall define invariants of $P,(\tau, T, \leqslant)$ as generalized Euler characteristics of $\mathrm{Obj}_{\mathrm{ss}}^{\alpha}(\tau)$, and study identities they satisfy, and transformation laws under change of stability condition.

To carry out this programme requires a theory of constructible sets and functions in algebraic $\mathbb{K}$-stacks, and compatible notions of Euler characteristic and pushforward. As I could not find these tools in the literature, I develop them here. It seemed better to write a stand-alone paper that others could use, rather than include the material in the series [8-11].

Section 2 gives some background on schemes, varieties and stacks. In 3 we recall MacPherson's constructible functions theory for $\mathbb{C}$-varieties, extend it to $\mathbb{K}$-schemes using l-adic cohomology in place of cohomology with the analytic topology for $\mathbb{C}$-varieties when $\mathbb{K}$ has characteristic zero, and define and 
study pseudomorphisms between locally constructible sets in $\mathbb{K}$-schemes. We also explain why the theory cannot be extended to $\mathbb{K}$-schemes for $\mathbb{K}$ of positive characteristic.

Sections 4 and 5 ] extend these ideas to stacks. An important difference between stacks and schemes is that in an algebraic $\mathbb{K}$-stack $\mathfrak{F}$ points $x \in \mathfrak{F}(\mathbb{K})$ have stabilizer groups $\operatorname{IsO}_{\mathbb{K}}(x)$, which are algebraic $\mathbb{K}$-groups, trivial if $\mathfrak{F}$ is a $\mathbb{K}$ scheme. It turns out that there are many different ways of including stabilizer groups when extending Euler characteristics $\chi$ and pushforwards CF to stacks.

We highlight three interesting cases, the naïve pushforward $\mathrm{CF}^{\text {na }}$ which ignores stabilizer groups, the stack pushforward $\mathrm{CF}^{\text {stk }}$ which is most natural in many stack problems, and the orbifold pushforward $\mathrm{CF}^{\text {orb }}$, related to DeligneMumford stacks and their crepant resolutions. Each is associated with a notion of Euler characteristic $\chi^{\text {na }}, \chi^{\text {stk }}, \chi^{\text {orb }}$ of constructible sets in $\mathbb{K}$-stacks.

As $\chi^{\text {stk }}, \mathrm{CF}^{\text {stk }}$ involve weighting by $1 / \chi\left(\operatorname{IsO}_{\mathbb{K}}(x)\right)$, the obvious definitions fail when $\chi\left(\operatorname{Iso}_{\mathbb{K}}(x)\right)=0$. However, for representable 1-morphisms $\phi: \mathfrak{F} \rightarrow \mathfrak{G}$ we give a more subtle definition of $\mathrm{CF}^{\mathrm{stk}}(\phi): \mathrm{CF}(\mathfrak{F}) \rightarrow \mathrm{CF}(\mathfrak{G})$ in 5.1 which is always well-defined, and suffices for the applications in [9-11]. We also define pullbacks $\psi^{*}$ by finite type 1-morphisms $\psi: \mathfrak{F} \rightarrow \mathfrak{G}$, and show pullbacks $\psi^{*}$ and pushforwards $\mathrm{CF}^{\text {stk }}(\phi)$ commute in Cartesian squares.

A companion paper [7] studies 'stack functions' on Artin stacks, which are a universal generalization of constructible functions containing more information, and discusses how 'motivic' invariants of $\mathbb{K}$-varieties such as Euler characteristics and virtual Poincaré polynomials are best extended to Artin stacks.

All $\mathbb{K}$-schemes and $\mathbb{K}$-stacks in this paper are assumed locally of finite type.

Acknowledgements. I thank Tom Bridgeland, Frances Kirwan, Andrew Kresch, Jörg Schürmann, Bertrand Toen and Burt Totaro for useful conversations. I held an EPSRC Advanced Research Fellowship whilst writing this paper.

\section{Schemes, varieties and stacks}

Fix an algebraically closed field $\mathbb{K}$ throughout. There are four main classes of 'spaces' over $\mathbb{K}$ used in algebraic geometry, in increasing order of generality:

$\mathbb{K}$-varieties $\subset \mathbb{K}$-schemes $\subset$ algebraic $\mathbb{K}$-spaces $\subset$ algebraic $\mathbb{K}$-stacks.

Section 2.1 gives a few definitions and facts on $\mathbb{K}$-schemes and $\mathbb{K}$-varieties, and 2.2 introduces algebraic $\mathbb{K}$-stacks. Some good references for 2.1 are Hartshorne [5], and for $\$ 2.2$ are Gómez [3] and Laumon and Moret-Bailly [15].

\subsection{Schemes and varieties}

We assume a good knowledge of $\mathbb{K}$-schemes and their morphisms, following Hartshorne [5]. We make the conventions that:

- All $\mathbb{K}$-schemes in this paper are locally of finite type.

- All $\mathbb{K}$-subschemes are locally closed, but not necessarily closed. 
- A $\mathbb{K}$-variety is a reduced, irreducible, separated $\mathbb{K}$-scheme of finite type.

Definition 2.1. For a $\mathbb{K}$-scheme $X$, write $X(\mathbb{K})$ for the set $\operatorname{Hom}(\operatorname{Spec} \mathbb{K}, X)$ of morphisms of $\mathbb{K}$-schemes Spec $\mathbb{K} \rightarrow X$. Then $X(\mathbb{K})$ is naturally identified with the subset of closed points of the underlying topological space of $X$. Elements of $X(\mathbb{K})$ are also called geometric points or $\mathbb{K}$-points of $X$.

There is a natural identification $(X \times Y)(\mathbb{K}) \cong X(\mathbb{K}) \times Y(\mathbb{K})$. If $\phi: X \rightarrow Y$ is a morphism of $\mathbb{K}$-schemes, composition Spec $\mathbb{K} \rightarrow X \stackrel{\phi}{\longrightarrow} Y$ gives a natural map $\phi_{*}: X(\mathbb{K}) \rightarrow Y(\mathbb{K})$. If $X$ is a $\mathbb{K}$-subscheme of $Y$ then $X(\mathbb{K}) \subseteq Y(\mathbb{K})$.

Much of the paper will involve cutting schemes or stacks into pieces. To do this we shall use two different notions of disjoint union.

Definition 2.2. Let $X$ be a $\mathbb{K}$ scheme, and $\left\{X_{i}: i \in I\right\}$ a family of $\mathbb{K}$ subschemes of $X$. We say that $X$ is the set-theoretic disjoint union of the $X_{i}$ for $i \in I$ if $X(\mathbb{K})=\coprod_{i \in I} X_{i}(\mathbb{K})$.

If $\left\{X_{i}: i \in I\right\}$ is a family of $\mathbb{K}$-schemes, we define the abstract disjoint union of the $X_{i}$ to be the $\mathbb{K}$-scheme $\left(X, \mathcal{O}_{X}\right)$, where $X$ is the disjoint union of the topological spaces $X_{i}$, and $\left.\mathcal{O}_{X}\right|_{X_{i}}=\mathcal{O}_{X_{i}}$. Then $X$ exists and is unique up to isomorphism, and the $X_{i}$ are open and closed $\mathbb{K}$-subschemes of $X$. Clearly, an abstract disjoint union is a set-theoretic disjoint union, but not necessarily vice versa. When we just say 'disjoint union' we mean set-theoretic disjoint union.

Here is a useful result of Rosenlicht [21], on the existence of quotients of varieties by algebraic groups.

Theorem 2.3. Let $\mathbb{K}$ be an algebraically closed field, $X$ a $\mathbb{K}$-variety, $G$ an algebraic $\mathbb{K}$-group, and $\rho: G \times X \rightarrow X$ an algebraic action of $G$ on $X$. Then there exists a dense, Zariski open subset $X^{\prime}$ of $X, a \mathbb{K}$-variety $Y$, and a surjective morphism $\pi: X^{\prime} \rightarrow Y$ inducing a bijection between $G$-orbits in $X^{\prime}$ and $\mathbb{K}$-points in $Y$, such that any $G$-invariant rational function on $X^{\prime}$ defined at $x \in X^{\prime}$ is the pull-back of a rational function on $Y$ defined at $\pi(x)$.

\section{$2.2 \quad$ Algebraic stacks}

Algebraic stacks (also known as Artin stacks) were introduced by Artin, generalizing Deligne-Mumford stacks. For a good introduction to algebraic stacks see Gómez [3], and for a thorough treatment see Laumon and Moret-Bailly [15]. As for schemes, we make the convention that all algebraic $\mathbb{K}$-stacks in this paper are locally of finite type, and $\mathbb{K}$-substacks are locally closed.

Algebraic $\mathbb{K}$-stacks form a 2-category. That is, we have objects which are $\mathbb{K}$-stacks $\mathfrak{F}, \mathfrak{G}$, and also two kinds of morphisms, 1-morphisms $\phi, \psi: \mathfrak{F} \rightarrow \mathfrak{G}$ between $\mathbb{K}$-stacks, and 2-morphisms $A: \phi \rightarrow \psi$ between 1-morphisms. An analogy to keep in mind is a 2-category of categories, where objects are categories, 1-morphisms are functors between the categories, and 2-morphisms are isomorphisms (natural transformations) between functors.

We define the set of $\mathbb{K}$-points of a stack. 
Definition 2.4. Let $\mathfrak{F}$ be a $\mathbb{K}$-stack. Write $\mathfrak{F}(\mathbb{K})$ for the set of 2 -isomorphism classes $[x]$ of 1-morphisms $x: \operatorname{Spec} \mathbb{K} \rightarrow \mathfrak{F}$. Elements of $\mathfrak{F}(\mathbb{K})$ are called $\mathbb{K}$-points, or geometric points, of $\mathfrak{F}$. If $\phi: \mathfrak{F} \rightarrow \mathfrak{G}$ is a 1-morphism then composition with $\phi$ induces a map of sets $\phi_{*}: \mathfrak{F}(\mathbb{K}) \rightarrow \mathfrak{G}(\mathbb{K})$.

For a 1-morphism $x: \operatorname{Spec} \mathbb{K} \rightarrow \mathfrak{F}$, the stabilizer group $\operatorname{Iso}_{\mathbb{K}}(x)$ is the group of 2-morphisms $x \rightarrow x$. When $\mathfrak{F}$ is an algebraic $\mathbb{K}$-stack, Iso $\mathbb{K}(x)$ is an algebraic $\mathbb{K}$-group. We say that $\mathfrak{F}$ has affine geometric stabilizers if $\operatorname{IsO}_{\mathbb{K}}(x)$ is an affine algebraic $\mathbb{K}$-group for all 1-morphisms $x: \operatorname{Spec} \mathbb{K} \rightarrow \mathfrak{F}$.

As an algebraic $\mathbb{K}$-group up to isomorphism, $\operatorname{Iso}_{\mathbb{K}}(x)$ depends only on the isomorphism class $[x] \in \mathfrak{F}(\mathbb{K})$ of $x$ in $\operatorname{Hom}(\operatorname{Spec} \mathbb{K}, \mathfrak{F})$. If $\phi: \mathfrak{F} \rightarrow \mathfrak{G}$ is a 1-morphism, composition induces a morphism of algebraic $\mathbb{K}$-groups $\phi_{*}$ : $\mathrm{IsO}_{\mathbb{K}}([x]) \rightarrow \operatorname{IsO}_{\mathbb{K}}\left(\phi_{*}([x])\right)$, for $[x] \in \mathfrak{F}(\mathbb{K})$.

One important difference in working with 2-categories rather than ordinary categories is that in diagram-chasing one only requires 1-morphisms to be 2isomorphic rather than equal. The simplest kind of commutative diagram is:

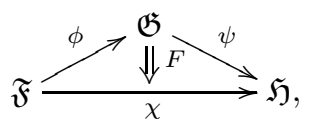

by which we mean that $\mathfrak{F}, \mathfrak{G}, \mathfrak{H}$ are $\mathbb{K}$-stacks, $\phi, \psi, \chi$ are 1 -morphisms, and $F$ : $\psi \circ \phi \rightarrow \chi$ is a 2 -isomorphism. Usually we omit $F$, and mean that $\psi \circ \phi \cong \chi$.

Definition 2.5. Let $\phi: \mathfrak{F} \rightarrow \mathfrak{H}, \psi: \mathfrak{G} \rightarrow \mathfrak{H}$ be 1-morphisms of $\mathbb{K}$-stacks. Then one can define the fibre product stack $\mathfrak{F} \times_{\phi, \mathfrak{H}, \psi} \mathfrak{G}$, or $\mathfrak{F} \times_{\mathfrak{H}} \mathfrak{G}$ for short, with 1-morphisms $\pi_{\mathfrak{F}}, \pi_{\mathfrak{G}}$ fitting into a commutative diagram:

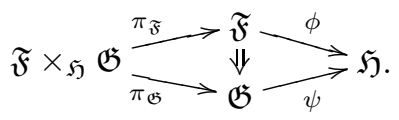

A commutative diagram

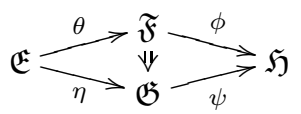

is a Cartesian square if it is isomorphic to (10), so there is a 1 -isomorphism $\mathfrak{E} \cong$ $\mathfrak{F} \times \mathfrak{H} \mathfrak{G}$. Cartesian squares may also be characterized by a universal property.

Here is a definition from Kresch [14, Def. 3.5.3], slightly modified.

Definition 2.6. Let $\mathfrak{F}$ be a finite type algebraic $\mathbb{K}$-stack, and $\mathfrak{F}^{\text {red }}$ the associated reduced stack. We say that $\mathfrak{F}$ can be stratified by global quotient stacks if $\mathfrak{F}^{\text {red }}$ is the disjoint union of finitely many locally closed substacks $U_{i}$ with each $U_{i}$ 1-isomorphic to a stack of the form $\left[X_{i} / G_{i}\right]$, where $X_{i}$ is a $\mathbb{K}$-variety and $G_{i}$ a smooth, connected, affine algebraic $\mathbb{K}$-group acting linearly on $X_{i}$. For a stack to be the disjoint union of a family of locally closed substacks is defined in $\left[15\right.$, p. 22]. It implies that $\mathfrak{F}(\mathbb{K})=\mathfrak{F}^{\text {red }}(\mathbb{K})=\bigsqcup_{i} U_{i}(\mathbb{K})$. 
Kresch [14, Prop. 3.5.2(ii)] takes the $X_{i}$ to be quasiprojective schemes, rather than varieties, but this is equivalent to our definition. Kresch [14, Prop. 3.5.9] characterizes stacks stratified by global quotients.

Theorem 2.7. Let $\mathfrak{F}$ be a finite type algebraic $\mathbb{K}$-stack. Then $\mathfrak{F}$ can be stratified by global quotient stacks if and only if $\mathfrak{F}$ has affine geometric stabilizers.

\section{Constructible functions on $\mathbb{K}$-schemes}

We now introduce constructible sets and functions on $\mathbb{K}$-schemes, and the pushforward of constructible functions by morphisms. Section 3.1 defines (locally) constructible sets and functions on $\mathbb{K}$-schemes. We explain the Euler characteristic and pushforwards over $\mathbb{C}$ in 3.2 and over other fields $\mathbb{K}$ in 3.3 Section 3.4 defines pseudomorphisms, a notion of morphism for (locally) constructible sets, and pushforwards along pseudomorphisms.

Some references are Mumford [19, p. 51] and Hartshorne [5, p. 94] for constructible sets, and MacPherson [16], Viro [22] and Kennedy [13] for constructible functions and the pushforward. As far as the author can tell the ideas of 3.3 3.4 are new, although elementary and probably obvious to experts.

\subsection{Constructible sets and functions on $\mathbb{K}$-schemes}

We define constructible and locally constructible sets.

Definition 3.1. Let $\mathbb{K}$ be an algebraically closed field, and $X$ a $\mathbb{K}$-scheme. A subset $C \subseteq X(\mathbb{K})$ is called constructible if $C=\bigcup_{i \in I} X_{i}(\mathbb{K})$, where $\left\{X_{i}: i \in I\right\}$ is a finite collection of finite type $\mathbb{K}$-subschemes $X_{i}$ of $X$. We call $S \subseteq X(\mathbb{K})$ locally constructible if $S \cap C$ is constructible for all constructible $C \subseteq X(\mathbb{K})$.

This is easily seen to be equivalent to a stronger definition, where we take the union $C=\bigcup_{i \in I} X_{i}(\mathbb{K})$ to be disjoint, and the $X_{i}$ to be separated.

Proposition 3.2. Let $X$ be a $\mathbb{K}$-scheme, and $C \subseteq X(\mathbb{K})$ a constructible subset. Then we may write $C=\bigsqcup_{i \in I} X_{i}(\mathbb{K})$, where $\left\{X_{i}: i \in I\right\}$ is a finite collection of separated, finite type $\mathbb{K}$-subschemes $X_{i}$ of $X$.

The following properties of constructible sets in $\mathbb{K}$-varieties are well known, [5, p. 94], [19, p. 51]. Our extension to $\mathbb{K}$-schemes is straightforward.

Proposition 3.3. Let $X, Y$ be $\mathbb{K}$-schemes, $\phi: X \rightarrow Y$ a morphism, and $A, B \subseteq$ $X(\mathbb{K})$ be constructible subsets. Then $A \cup B, A \cap B$ and $A \backslash B$ are constructible in $X(\mathbb{K})$, and $\phi_{*}(A)$ is constructible in $Y(\mathbb{K})$.

Note that showing $\phi_{*}(A)$ constructible, and the stack analogue in Proposition 4.5] are the only places we use the convention that $\mathbb{K}$-schemes and $\mathbb{K}$-stacks are locally of finite type. Next we define (locally) constructible functions. 
Definition 3.4. Let $X$ be a $\mathbb{K}$-scheme and $S \subseteq X(\mathbb{K})$ be locally constructible. A constructible function on $S$ is a function $f: S \rightarrow \mathbb{Q}$ such that $f(S)$ is finite and $f^{-1}(c)$ is a constructible set in $S \subseteq X(\mathbb{K})$ for each $c \in f(S) \backslash\{0\}$. Note that we do not require $f^{-1}(0)$ to be constructible. Write $\mathrm{CF}(S)$ for the $\mathbb{Q}$-vector space of constructible functions on $S$, and for brevity write $\mathrm{CF}(X)$ for $\mathrm{CF}(X(\mathbb{K}))$.

A locally constructible function on $S$ is a function $f: S \rightarrow \mathbb{Q}$ such that $\left.f\right|_{C}$ is constructible for all constructible $C \subseteq S$. Equivalently, $f$ is locally constructible if $f^{-1}(c)$ is locally constructible for all $c \in \mathbb{Q}$, and $f(C)$ is finite for all constructible $C \subseteq S$. Write $\operatorname{LCF}(S)$ for the $\mathbb{Q}$-vector space of locally constructible functions on $S$, and $\operatorname{LCF}(X)$ for $\operatorname{LCF}(X(\mathbb{K}))$.

Using Proposition 3.3 we see that products of (locally) constructible functions are (locally) constructible, so $\mathrm{CF}(S)$ and $\operatorname{LCF}(S)$ are commutative $\mathbb{Q}$ algebras, with $\mathrm{CF}(S)$ an ideal in $\operatorname{LCF}(S)$. Note that $1 \in \mathrm{CF}(S)$ if and only if $S$ is constructible, so if it is not then $\mathrm{CF}(S)$ is an algebra without identity.

Here are some remarks on this material:

- To define constructible functions $f: X(\mathbb{K}) \rightarrow \mathbb{Q}$ on $\mathbb{K}$-schemes $X$ which are not of finite type, or $f: S \rightarrow \mathbb{Q}$ for $S$ not constructible, we must allow $f^{-1}(0)$ to be non-constructible. If we did not there would be no constructible functions on $X$ or $S$, not even 0 .

For $X$ not of finite type we can think of $X(\mathbb{K})$ as being 'large', or 'unbounded'. Constructible functions $f: X(\mathbb{K}) \rightarrow \mathbb{Q}$ are nonzero only on small, bounded subsets of $X(\mathbb{K})$, and $f^{-1}(0)$ is the remaining, large, unbounded part of $X(\mathbb{K})$.

- We can also consider constructible functions with values in $\mathbb{Z}$, or any other abelian group, ring or field. But for simplicity we restrict to $\mathbb{Q}$.

\subsection{Euler characteristics and pushforward for $\mathbb{C}$-schemes}

We define the analytic Euler characteristic $\chi_{\text {an }}$.

Definition 3.5. Let $X$ be a separated $\mathbb{C}$-scheme of finite type. Then $X(\mathbb{C})$ is a Hausdorff topological space with the analytic topology. Write $\chi_{\text {an }}(X)$ for the Euler characteristic of $X(\mathbb{C})$, in compactly-supported cohomology.

The following properties of $\chi_{\text {an }}$ are well known.

Proposition 3.6. Let $X, Y$ be separated $\mathbb{C}$-schemes of finite type. Then

(i) If $Z$ is a closed subscheme of $X$ then $\chi_{\mathrm{an}}(X)=\chi_{\mathrm{an}}(Z)+\chi_{\mathrm{an}}(X \backslash Z)$.

(ii) Suppose $X$ is the set-theoretic disjoint union of subschemes $U_{1}, \ldots, U_{m}$. Then $\chi_{\mathrm{an}}(X)=\sum_{i=1}^{m} \chi_{\mathrm{an}}\left(U_{m}\right)$.

(iii) $\chi_{\mathrm{an}}(X \times Y)=\chi_{\mathrm{an}}(X) \chi_{\mathrm{an}}(Y)$.

(iv) If $\phi: X \rightarrow Y$ is a morphism which is a locally trivial fibration in the analytic topology with fibre $F$, then $\chi_{\mathrm{an}}(X)=\chi_{\mathrm{an}}(F) \chi_{\mathrm{an}}(Y)$.

(v) $\chi_{\text {an }}\left(\mathbb{C}^{m}\right)=1$ and $\chi_{\text {an }}\left(\mathbb{C P}^{m}\right)=m+1$ for all $m \geqslant 0$. 
Now we can define pushforwards on $\mathbb{C}$-schemes.

Definition 3.7. Let $X$ be a $\mathbb{C}$-scheme and $C \subseteq X(\mathbb{C})$ a constructible subset. Proposition 3.2 gives $C=\coprod_{i \in I} X_{i}(\mathbb{C})$ for $\left\{X_{i}: i \in I\right\}$ finitely many separated, finite type subschemes of $X$. Define $\chi_{\text {an }}(C)=\sum_{i \in I} \chi_{\text {an }}\left(X_{i}\right)$. If $\left\{Y_{j}: j \in J\right\}$ is another choice from Proposition 3.2 then $X_{i}$ is the set-theoretic union of $X_{i} \cap Y_{j}$ for $j \in J$, so Proposition 3.6(ii) gives $\chi_{\text {an }}\left(X_{i}\right)=\sum_{j \in J} \chi_{\text {an }}\left(X_{i} \cap Y_{j}\right)$. Hence

$$
\sum_{i \in I} \chi_{\mathrm{an}}\left(X_{i}\right)=\sum_{i \in I}\left[\sum_{j \in J} \chi_{\mathrm{an}}\left(X_{i} \cap Y_{j}\right)\right]=\sum_{j \in J}\left[\sum_{i \in I} \chi_{\mathrm{an}}\left(X_{i} \cap Y_{j}\right)\right]=\sum_{j \in J} \chi_{\mathrm{an}}\left(Y_{j}\right),
$$

and $\chi_{\mathrm{an}}(C)$ is well-defined.

For $f \in \mathrm{CF}(X)$, define the weighted Euler characteristic $\chi_{\text {an }}(X, f) \in \mathbb{Q}$ by

$$
\chi_{\mathrm{an}}(X, f)=\sum_{c \in f(X(\mathbb{C})) \backslash\{0\}} c \chi_{\mathrm{an}}\left(f^{-1}(c)\right) .
$$

This is well-defined as $f(X(\mathbb{C}))$ is finite and $f^{-1}(c) \subseteq X(\mathbb{C})$ is constructible for each $c \in f(X(\mathbb{C})) \backslash\{0\}$. Clearly, $f \mapsto \chi_{\text {an }}(X, f)$ is a linear map $\mathrm{CF}(X) \rightarrow \mathbb{Q}$.

Now let $\phi: X \rightarrow Y$ be a morphism of $\mathbb{C}$-schemes, and $f \in \operatorname{CF}(X)$. Define the pushforward $\mathrm{CF}(\phi) f: Y(\mathbb{C}) \rightarrow \mathbb{Q}$ of $f$ to $Y$ by

$$
\mathrm{CF}(\phi) f(y)=\chi_{\mathrm{an}}\left(X, f \cdot \delta_{\phi_{*}^{-1}(y)}\right) \quad \text { for } y \in Y(\mathbb{C}) .
$$

Here $\phi_{*}: X(\mathbb{C}) \rightarrow Y(\mathbb{C})$ is the induced map, $\phi_{*}^{-1}(y) \subseteq X(\mathbb{C})$ is the inverse image of $\{y\}$ under $\phi_{*}$, and $\delta_{\phi_{*}^{-1}(y)}$ is its characteristic function. It is a locally constructible function, so $f \cdot \delta_{\phi_{*}^{-1}(y)} \in \mathrm{CF}(X)$, and (3) is well-defined.

MacPherson [16, Prop. 1] gives an important property of the pushforward for algebraic $\mathbb{C}$-varieties. The extension to $\mathbb{C}$-schemes is straightforward. One can prove it by dividing $X, Y$ into pieces upon which $\phi$ is a locally trivial fibration in the analytic topology, and using Proposition 3.6(ii),(iv).

Theorem 3.8. Let $X, Y, Z$ be $\mathbb{C}$-schemes, $\phi: X \rightarrow Y$ and $\psi: Y \rightarrow Z$ be morphisms, and $f \in \mathrm{CF}(X)$. Then $\mathrm{CF}(\phi) f$ is constructible, so $\operatorname{CF}(\phi): \operatorname{CF}(X) \rightarrow$ $\mathrm{CF}(Y)$ is a $\mathbb{Q}$-linear map. Also $\mathrm{CF}(\psi \circ \phi)=\mathrm{CF}(\psi) \circ \mathrm{CF}(\phi)$ as linear maps $\mathrm{CF}(X) \rightarrow \mathrm{CF}(Z)$. Hence $\mathrm{CF}$ is a functor from the category of $\mathbb{C}$-schemes to the category of $\mathbb{Q}$-vector spaces.

Viro [22] gives an interesting point of view on constructible functions. One can regard the Euler characteristic as a measure, defined on constructible sets. Then $\chi_{\mathrm{an}}(X, f)$ in (2) is the integral of $f$ with respect to this measure, and the pushforward $\mathrm{CF}(\phi) f$ integrates $f$ over the fibres of $\phi$.

\subsection{Extension to other fields $\mathbb{K}$}

To extend 3.2 to other fields $\mathbb{K}$, we need a good notion of Euler characteristic $\chi(X)$ for a separated $\mathbb{K}$-scheme $X$ of finite type. 
Definition 3.9. Let $\mathbb{K}$ be an algebraically closed field of characteristic $p$, which may be zero, and fix a prime number $l \neq p$. Write $\mathbb{Q}_{l}$ for the field of l-adic rationals. Let $X$ be a separated $\mathbb{K}$-scheme of finite type. Then one may define the compactly-supported l-adic cohomology groups $H_{\mathrm{cs}}^{i}\left(X, \mathbb{Q}_{l}\right)$ of $X$, for $i \geqslant 0$. The original reference for étale and $l$-adic cohomology is Grothendieck et al. [4], and a good book is Milne [18]. Define the Euler characteristic $\chi(X)$ of $X$ to be

$$
\chi(X)=\sum_{i=0}^{2 \operatorname{dim} X}(-1)^{i} \operatorname{dim}_{\mathbb{Q}_{l}} H_{\mathrm{cs}}^{i}\left(X, \mathbb{Q}_{l}\right) .
$$

Here are some properties of $\chi$, generalizing Proposition 3.6.

Theorem 3.10. Let $\mathbb{K}$ be an algebraically closed field and $X, Y$ be separated $\mathbb{K}$-schemes of finite type. Then

(i) If $Z$ is a closed subscheme of $X$ then $\chi(X)=\chi(Z)+\chi(X \backslash Z)$.

(ii) Suppose $X$ is the set-theoretic disjoint union of subschemes $U_{1}, \ldots, U_{m}$. Then $\chi(X)=\sum_{i=1}^{m} \chi\left(U_{i}\right)$.

(iii) $\chi(X \times Y)=\chi(X) \chi(Y)$.

(iv) $\chi(X)$ is independent of the choice of $l$ in Definition 3.9

(v) When $\mathbb{K}=\mathbb{C}$ we have $\chi(X)=\chi \mathrm{an}(X)$.

(vi) $\chi\left(\mathbb{K}^{m}\right)=1$ and $\chi\left(\mathbb{K} \mathbb{P}^{m}\right)=m+1$ for all $m \geqslant 0$.

Proof. Part (i) comes from the long exact sequence [4, 4.XVII.5.1.16]:

$$
\cdots \rightarrow H_{\mathrm{cs}}^{i}\left(X \backslash Z, \mathbb{Q}_{l}\right) \rightarrow H_{\mathrm{cs}}^{i}\left(X, \mathbb{Q}_{l}\right) \rightarrow H_{\mathrm{cs}}^{i}\left(Z, \mathbb{Q}_{l}\right) \rightarrow H_{\mathrm{cs}}^{i+1}\left(X \backslash Z, \mathbb{Q}_{l}\right) \rightarrow \cdots,
$$

and (ii) follows from (i) by standard arguments. Part (iii) is a consequence of the Künneth formula [4, 4.XVII.5.4.3]. Part (iv) is proved for $X$ proper in [4, 5.VII.4.10]. The general case follows from (i) as we may write $X \cong \bar{X} \backslash Z$ for $\bar{X}$ a proper separated $\mathbb{K}$-scheme of finite type, and $Z$ a closed subscheme. Part (v) follows from the comparison theorem [4, 4.XVI.4.1]. For (vi), calculation shows $H_{\mathrm{cs}}^{i}\left(\mathbb{K}^{m}, \mathbb{Q}_{l}\right)$ is $\mathbb{Q}_{l}$ if $i=2 m$ and 0 otherwise, so $\chi\left(\mathbb{K}^{m}\right)=1$. Then $\chi\left(\mathbb{K} \mathbb{P}^{m}\right)=m+1$ by (ii) and $\mathbb{K} \mathbb{P}^{m}=\coprod_{n=0}^{m} \mathbb{K}^{n}$.

Here are the generalizations of Definition 3.7 and Theorem 3.8 to $\mathbb{K}$.

Definition 3.11. Let $X$ be a $\mathbb{K}$-scheme and $C \subseteq X(\mathbb{K})$ a constructible subset. Write $C=\coprod_{i \in I} X_{i}(\mathbb{K})$ as in Proposition 3.2 and define $\chi(C)=\sum_{i \in I} \chi\left(X_{i}\right)$. This is well-defined as in Definition 3.7 using Theorem 3.10(ii). For $f \in \mathrm{CF}(X)$, define the weighted Euler characteristic $\chi(X, f) \in \mathbb{Q}$ by

$$
\chi(X, f)=\sum_{c \in f(X(\mathbb{K})) \backslash\{0\}} c \chi\left(f^{-1}(c)\right) .
$$

Then $f \mapsto \chi(X, f)$ is a linear map $\mathrm{CF}(X) \rightarrow \mathbb{Q}$. Let $\phi: X \rightarrow Y$ be a morphism of $\mathbb{K}$-schemes. Define the pushforward $\mathrm{CF}(\phi) f: Y(\mathbb{K}) \rightarrow \mathbb{Q}$ of $f$ to $Y$ by

$$
\mathrm{CF}(\phi) f(y)=\chi\left(X, f \cdot \delta_{\phi_{*}^{-1}(y)}\right) \quad \text { for } y \in Y(\mathbb{K}) .
$$

When $\mathbb{K}=\mathbb{C}$ these definitions agree with Definition 3.7 by Theorem 3.10(v). 
There are several ways to prove the next theorem. One is to use results of Kennedy [13]. He defines pushforwards implicitly using intersections of Lagrangian cycles, but one can show using base change and comparison theorems for $l$-adic cohomology that his definition of $\mathrm{CF}(\phi)$ agrees with ours. Another is to use Katz and Laumon [12, Th. 3.1.2], which in characteristic zero relates pushforwards of constructible sheaves and functions, so functoriality of CF follows from that for sheaf pushforwards.

Theorem 3.12. Let $\mathbb{K}$ be an algebraically closed field of characteristic zero, $X, Y, Z$ be $\mathbb{K}$-schemes, $\phi: X \rightarrow Y$ and $\psi: Y \rightarrow Z$ be morphisms, and $f \in$ $\mathrm{CF}(X)$. Then $\mathrm{CF}(\phi) f$ is constructible, so $\mathrm{CF}(\phi): \mathrm{CF}(X) \rightarrow \mathrm{CF}(Y)$ is a $\mathbb{Q}$ linear map. Also $\mathrm{CF}(\psi \circ \phi)=\mathrm{CF}(\psi) \circ \mathrm{CF}(\phi)$, so $\mathrm{CF}$ is a functor.

The last part of the theorem is false for $\mathbb{K}$ of characteristic $p>0$, and I am grateful to Jörg Schürmann for the following explanation of why. The proof using constructible sheaves fails because if $\mathcal{L}$ is a locally constant $\mathbb{Q}_{l}$-sheaf of rank $r$ on a non-proper $\mathbb{K}$-scheme $X$ for $l \neq p$, we need the fact that

$$
\chi\left(H_{\mathrm{cs}}^{*}(X, \mathcal{L})\right)=r \cdot \chi\left(H_{\mathrm{cs}}^{*}\left(X, \mathbb{Q}_{l}\right)\right) .
$$

This holds in characteristic zero, but not in characteristic $p>0$ without extra conditions on $\mathcal{L}$, which are studied in Illusie [6].

Here is a counterexample to Theorem $\mathbf{3 . 1 2}$ in positive characteristic. Let $\mathbb{K}$ have characteristic $p \geqslant 2$, and $\phi: \mathbb{K} \rightarrow \mathbb{K}$ be the Artin-Schreier morphism $\phi: x \mapsto x^{p}-x$. It is a $p$-fold étale covering of $\mathbb{K}$ by itself, so $\mathrm{CF}(\phi) 1=p$ in $\mathrm{CF}(\mathbb{K})$. Thus taking $\psi: \mathbb{K} \rightarrow \operatorname{Spec} \mathbb{K}$ to be the projection we have $\mathrm{CF}(\psi \circ \phi) 1=1$ but $\mathrm{CF}(\psi) \circ \mathrm{CF}(\phi) 1=p$ in $\mathrm{CF}(\operatorname{Spec} \mathbb{K})=\mathbb{Q}$, so $\mathrm{CF}(\psi \circ \phi) \neq \mathrm{CF}(\psi) \circ \mathrm{CF}(\phi)$.

When $Z=\operatorname{Spec} \mathbb{K}$ and $\psi: Y \rightarrow \operatorname{Spec} \mathbb{K}$ is the projection we have $\operatorname{CF}(Z)=\mathbb{Q}$ and $\mathrm{CF}(\psi) g=\chi(Y, g)$ for $g \in \mathrm{CF}(Y)$. So $\mathrm{CF}(\psi \circ \phi)=\mathrm{CF}(\psi) \circ \mathrm{CF}(\phi)$ gives a relation between pushforwards and weighted Euler characteristics:

Corollary 3.13. Let $\mathbb{K}$ have characteristic zero, $X, Y$ be $\mathbb{K}$-schemes, $\phi: X \rightarrow$ $Y$ a morphism, and $f \in \mathrm{CF}(X)$. Then $\chi(X, f)=\chi(Y, \mathrm{CF}(\phi) f)$.

\subsection{Extension to pseudomorphisms}

We define pseudomorphisms, a notion of morphism between locally constructible sets that generalizes morphisms of schemes.

Definition 3.14. Suppose $\mathbb{K}$ is an algebraically closed field, $X, Y$ are $\mathbb{K}$-schemes and $S \subseteq X(\mathbb{K}), T \subseteq Y(\mathbb{K})$ are locally constructible. Let $\Phi: S \rightarrow T$ be a map, and define the graph $\Gamma_{\Phi}=\{(s, \Phi(s)): s \in S\}$ in $X(\mathbb{K}) \times Y(\mathbb{K})=$ $(X \times Y)(\mathbb{K})$. We call $\Phi$ a pseudomorphism if $\Gamma_{\Phi} \cap(C \times Y(\mathbb{K}))$ is constructible for all constructible $C \subseteq X(\mathbb{K})$. This implies $\Gamma_{\Phi}$ is locally constructible.

A pseudomorphism $\Phi$ is a pseudoisomorphism if $\Phi$ is bijective and $\Phi^{-1}$ : $T \rightarrow S$ is a pseudomorphism. When $S=X(\mathbb{K})$ and $T=Y(\mathbb{K})$ we shall also call $\Phi: X \rightarrow Y$ a pseudomorphism (pseudoisomorphism) from $X$ to $Y$. 
When $X, Y$ are $\mathbb{K}$-varieties, pseudomorphisms $\Phi: X \rightarrow Y$ coincide with definable functions in the model theory of algebraic geometry. See for instance Marker [17, §7.4], in particular [17, Lem. 7.4.7] which shows that $\Phi$ equals a quasimorphism of varieties on a nonempty open affine subset $X_{0}$ of $X$, and a morphism if $\mathbb{K}$ has characteristic zero. Here are some basic properties of pseudomorphisms. They are easily proved using Proposition 3.3 and the projection morphisms $X \times Y \rightarrow Y, X \times Y \times Z \rightarrow X \times Z$.

Proposition 3.15. Let $\mathbb{K}$ be an algebraically closed field.

(a) Let $\phi: X \rightarrow Y$ be a morphism (isomorphism) of $\mathbb{K}$-schemes. Then $\phi_{*}: X(\mathbb{K}) \rightarrow Y(\mathbb{K})$ is a pseudomorphism (pseudoisomorphism).

(b) Let $X, Y$ be $\mathbb{K}$-schemes, $S \subseteq X(\mathbb{K}), T \subseteq Y(\mathbb{K})$ be locally constructible, $\Phi: S \rightarrow T$ be a pseudomorphism, and $C \subseteq S$ be constructible. Then $\Phi(C)$ is constructible in $Y(\mathbb{K})$. Also, if $t \in T$ then $C \cap \Phi^{-1}(t)$ is constructible in $X(\mathbb{K})$. Hence, $\Phi^{-1}(t)$ is locally constructible in $X(\mathbb{K})$.

(c) Let $X, Y, Z$ be $\mathbb{K}$-schemes, $S \subseteq X(\mathbb{K}), T \subseteq Y(\mathbb{K}), U \subseteq Z(\mathbb{K})$ be locally constructible, and $\Phi: S \rightarrow T, \bar{\Psi}: T \rightarrow U$ be pseudo(iso)morphisms. Then $\Psi \circ \Phi: S \rightarrow U$ is a pseudo(iso)morphism.

We define pushforwards $\mathrm{CF}(\Phi): \mathrm{CF}(S) \rightarrow \mathrm{CF}(T)$ along pseudomorphisms.

Definition 3.16. Let $X, Y$ be $\mathbb{K}$-schemes, $S \subseteq X(\mathbb{K}), T \subseteq Y(\mathbb{K})$ be locally constructible, $\Phi: S \rightarrow T$ a pseudomorphism, and $f \in \mathrm{CF}(S)$. Define the pushforward $\mathrm{CF}(\Phi) f: T \rightarrow \mathbb{Q}$ by

$$
\mathrm{CF}(\Phi) f(t)=\chi\left(S, f \cdot \delta_{\Phi^{-1}(t)}\right) \quad \text { for } t \in T .
$$

Here $\delta_{\Phi^{-1}(t)}$ is the characteristic function of $\Phi^{-1}(t) \subseteq S$ on $S$. By Proposition 3.15 (b) $\delta_{\Phi^{-1}(t)} \in \mathrm{LCF}(S)$, and $f \in \mathrm{CF}(S)$, so $f \cdot \delta_{\Phi^{-1}(t)} \in \mathrm{CF}(S)$. Thus (7) is well-defined, by Definition 3.11. If $\phi: X \rightarrow Y$ is a morphism of $\mathbb{K}$-schemes then $\phi_{*}: X(\mathbb{K}) \rightarrow Y(\mathbb{K})$ is a pseudomorphism by Proposition 3.15(a), and $\mathrm{CF}(\phi)$ in Definition 3.11 coincides with $\mathrm{CF}\left(\phi_{*}\right)$ above.

Here is the generalization of Theorems 3.8 and 3.12 to pseudomorphisms.

Theorem 3.17. Let $\mathbb{K}$ have characteristic zero, $X, Y, Z$ be $\mathbb{K}$-schemes, $S \subseteq$ $X(\mathbb{K}), T \subseteq Y(\mathbb{K}), U \subseteq Z(\mathbb{K})$ be locally constructible, $\Phi: S \rightarrow T, \Psi: T \rightarrow U$ be pseudomorphisms, and $f \in \mathrm{CF}(S)$. Then $\mathrm{CF}(\Phi) f$ is constructible, so $\mathrm{CF}(\Phi)$ : $\mathrm{CF}(S) \rightarrow \mathrm{CF}(T)$ is $\mathbb{Q}$-linear, and $\mathrm{CF}(\Psi \circ \Phi)=\mathrm{CF}(\Psi) \circ \mathrm{CF}(\Phi)$.

Proof. Define $F_{X Y}: X(\mathbb{K}) \times Y(\mathbb{K}) \rightarrow \mathbb{Q}, F_{X Z}: X(\mathbb{K}) \times Z(\mathbb{K}) \rightarrow \mathbb{Q}, F_{Y Z}: Y(\mathbb{K}) \times$ 


$$
\begin{aligned}
Z(\mathbb{K}) \rightarrow \mathbb{Q} \text { and } F_{X Y Z}: X(\mathbb{K}) \times Y(\mathbb{K}) \times Z(\mathbb{K}) \rightarrow \mathbb{Q} \text { by } \\
F_{X Y}(x, y)= \begin{cases}f(x), & x \in S \text { and } y=\Phi(x), \\
0, & \text { otherwise }\end{cases} \\
F_{X Z}(x, z)= \begin{cases}f(x), & x \in S \text { and } z=\Psi \circ \Phi(x), \\
0, & \text { otherwise, }\end{cases} \\
F_{Y Z}(y, z)= \begin{cases}(\mathrm{CF}(\Phi) f)(y), & y \in T \text { and } z=\Psi(y), \\
0, & \text { otherwise. }\end{cases} \\
F_{X Y Z}(x, y, z)= \begin{cases}f(x), & x \in S, y=\Phi(x) \text { and } z=\Psi(y) \\
0, & \text { otherwise. }\end{cases}
\end{aligned}
$$

Write $\Pi_{Y}^{X Y}: X \times Y \rightarrow Y$ for the projection morphism, and so on. It is easy to show $F_{X Y} \in \mathrm{CF}(X \times Y)$, so $\mathrm{CF}\left(\Pi_{Y}^{X Y}\right) F_{X Y} \in \mathrm{CF}(Y)$ by Theorem 3.12 But comparing (6) - (8) shows $\mathrm{CF}(\Phi) f=\left.\left(\mathrm{CF}\left(\Pi_{Y}^{X Y}\right) F_{X Y}\right)\right|_{T}$. Therefore $\mathrm{CF}(\Phi) f \in \mathrm{CF}(T)$, proving the first part. For the second part, $F_{X Z}, F_{Y Z}, F_{X Y Z}$ are constructible on $X \times Z, Y \times Z$ and $X \times Y \times Z$ in the same way, and it is easy to prove that

$$
\begin{aligned}
& \mathrm{CF}(\Psi \circ \Phi) f=\left.\left(\mathrm{CF}\left(\Pi_{Z}^{X Z}\right) F_{X Z}\right)\right|_{U}, \quad \mathrm{CF}(\Psi) \circ \mathrm{CF}(\Phi) f=\left.\left(\mathrm{CF}\left(\Pi_{Z}^{Y Z}\right) F_{Y Z}\right)\right|_{U}, \\
& \mathrm{CF}\left(\Pi_{X Z}^{X Y Z}\right) F_{X Y Z}=F_{X Z} \quad \text { and } \quad \mathrm{CF}\left(\Pi_{Y Z}^{X Y Z}\right) F_{X Y Z}=F_{Y Z} \text {. }
\end{aligned}
$$

But as $\Pi_{Z}^{X Z} \circ \Pi_{X Z}^{X Y Z}=\Pi_{Z}^{X Y Z}=\Pi_{Z}^{Y Z} \circ \Pi_{Y Z}^{X Y Z}$ Theorem 3.12 gives $\mathrm{CF}\left(\Pi_{Z}^{X Z}\right) \circ$ $\mathrm{CF}\left(\Pi_{X Z}^{X Y Z}\right)=\mathrm{CF}\left(\Pi_{Z}^{X Y Z}\right)=\mathrm{CF}\left(\Pi_{Z}^{Y Z}\right) \circ \mathrm{CF}\left(\Pi_{Y Z}^{X Y Z}\right)$, and the result follows.

If $\Phi: S \rightarrow T$ is a pseudoisomorphism then $\Phi^{-1}(t)$ is a single point in (77), giving $\mathrm{CF}(\Phi) f(t)=f \circ \Phi^{-1}(t)$. We deduce:

Corollary 3.18. Let $X, Y$ be $\mathbb{K}$-schemes, $S \subseteq X(\mathbb{K}), T \subseteq Y(\mathbb{K})$ be locally constructible, and $\Phi: S \rightarrow T$ a pseudoisomorphism. Then $\operatorname{CF}(\Phi): \operatorname{CF}(S) \rightarrow$ $\mathrm{CF}(T)$ is an isomorphism, with $\mathrm{CF}(\Phi) f=f \circ \Phi^{-1}$ and $\mathrm{CF}\left(\Phi^{-1}\right) g=g \circ \Phi$.

The moral is that pseudoisomorphic (locally) constructible sets are essentially the same from the point of view of constructible functions. So in problems involving constructible functions, we can work with (locally) constructible sets up to pseudoisomorphism, and pseudomorphisms between them.

\section{Constructible functions on stacks}

We now generalize $\$$ to stacks. Sections 4.1 and 4.2 develop the basic definitions and properties of constructible sets and functions, and show that any finite type algebraic $\mathbb{K}$-stack $\mathfrak{F}$ with affine geometric stabilizers is pseudoisomorphic to a finite type $\mathbb{K}$-scheme. This enables us to reduce to the scheme case of 93

An important difference between stacks and schemes is that points $x \in \mathfrak{F}(\mathbb{K})$ in a $\mathbb{K}$-stack $\mathfrak{F}$ have stabilizer groups $\operatorname{Iso}_{\mathbb{K}}(x)$, which are trivial if $\mathfrak{F}$ is a $\mathbb{K}$-scheme. There are many different ways of including stabilizer groups when extending 
Euler characteristics $\chi$ and pushforwards CF to stacks. Section 4.3 studies the simplest of these, the naïve versions $\chi^{\text {na }}, \mathrm{CF}^{\text {na }}$, which just ignore stabilizer groups. Given an allowable weight function $w$ upon affine algebraic $\mathbb{K}$-groups, in 44.4 we modify $\chi^{\text {na }}, \mathrm{CF}^{\text {na }}$ to get $\chi_{w}, \mathrm{CF}_{w}$ by weighting by $w_{\mathfrak{F}}: x \mapsto w\left(\operatorname{IsO}_{\mathbb{K}}(x)\right)$ on $\mathfrak{F}(\mathbb{K})$. Two special cases are the stack versions $\chi^{\text {stk }}, \mathrm{CF}^{\mathrm{stk}}$ which are most natural in many problems, and the orbifold versions $\chi^{\text {orb }}, \mathrm{CF}^{\text {orb }}$, related to Deligne-Mumford stacks and their crepant resolutions.

\subsection{Basic definitions}

We begin by giving analogues for stacks of the major definitions of 3 ,

Definition 4.1. Let $\mathbb{K}$ be an algebraically closed field, and $\mathfrak{F}$ an algebraic $\mathbb{K}$ stack. We call $C \subseteq \mathfrak{F}(\mathbb{K})$ constructible if $C=\bigcup_{i \in I} \mathfrak{F}_{i}(\mathbb{K})$, where $\left\{\mathfrak{F}_{i}: i \in I\right\}$ is a finite collection of finite type algebraic $\mathbb{K}$-substacks $\mathfrak{F}_{i}$ of $\mathfrak{F}$. We call $S \subseteq \mathfrak{F}(\mathbb{K})$ locally constructible if $S \cap C$ is constructible for all constructible $C \subseteq \mathfrak{F}(\mathbb{K})$.

Here is a partial analogue of Proposition 3.3 proved in the same way.

Lemma 4.2. Let $\mathfrak{F}$ be an algebraic $\mathbb{K}$-stack and $A, B \subseteq \mathfrak{F}(\mathbb{K})$ constructible subsets. Then $A \cup B, A \cap B$ and $A \backslash B$ are constructible in $\mathfrak{F}(\mathbb{K})$.

Definition 4.3. Let $\mathbb{K}$ be an algebraically closed field, $\mathfrak{F}$ an algebraic $\mathbb{K}$-stack, and $S \subseteq \mathfrak{F}(\mathbb{K})$ be locally constructible. Call a function $f: S \rightarrow \mathbb{Q}$ constructible if $f(S)$ is finite and $f^{-1}(c)$ is a constructible set for each $c \in f(S) \backslash\{0\}$. Call $f: S \rightarrow \mathbb{Q}$ locally constructible if $\left.f\right|_{C}$ is constructible for all constructible $C \subseteq$ $S \subseteq \mathfrak{F}(\mathbb{K})$. Write $\mathrm{CF}(S), \operatorname{LCF}(S)$ for the sets of (locally) constructible functions on $S$. Using Lemma 4.2 we see that $\operatorname{CF}(S), \operatorname{LCF}(S)$ are $\mathbb{Q}$-vector spaces. For brevity write $\mathrm{CF}(\mathfrak{F}), \operatorname{LCF}(\mathfrak{F})$ rather than $\mathrm{CF}(\mathfrak{F}(\mathbb{K})), \operatorname{LCF}(\mathfrak{F}(\mathbb{K}))$.

As in Definition 3.4 using Lemma 4.2 we see that multiplication of functions makes $\mathrm{CF}(S), \operatorname{LCF}(S)$ into commutative $\mathbb{Q}$-algebras, with $\mathrm{CF}(S)$ an ideal in $\mathrm{LCF}(S)$, and $\mathrm{CF}(S)$ is an algebra without identity if $S$ is not constructible.

Now let $\mathfrak{F}, \mathfrak{G}$ be algebraic $\mathbb{K}$-stacks, and $S \subseteq \mathfrak{F}(\mathbb{K}), T \subseteq \mathfrak{G}(\mathbb{K})$ be locally constructible. Then $\mathfrak{F} \times \mathfrak{G}$ is an algebraic $\mathbb{K}$-stack with $(\mathfrak{F} \times \mathfrak{G})(\mathbb{K})=\mathfrak{F}(\mathbb{K}) \times$ $\mathfrak{G}(\mathbb{K})$. Let $\Phi: S \rightarrow T$ be a map, and define the graph $\Gamma_{\Phi}=\{(s, \Phi(s)): s \in S\}$. We call $\Phi$ a pseudomorphism if $\Gamma_{\Phi} \cap(C \times \mathfrak{G}(\mathbb{K}))$ is constructible in $(\mathfrak{F} \times \mathfrak{G})(\mathbb{K})$ for all constructible $C \subseteq \mathfrak{F}(\mathbb{K})$. A pseudomorphism $\Phi$ is a pseudoisomorphism if $\Phi$ is bijective and $\Phi^{-1}: T \rightarrow S$ is a pseudomorphism.

These definitions agree with those of $₫ 3$ when $\mathfrak{F}, \mathfrak{G}$ are $\mathbb{K}$-schemes.

\subsection{Constructible sets and pseudomorphisms in stacks}

We now extend properties of constructible sets and pseudomorphisms in $\mathbb{K}$ schemes to algebraic $\mathbb{K}$-stacks with affine geometric stabilizers.

Proposition 4.4. Let $\mathfrak{F}$ be a finite type algebraic $\mathbb{K}$-stack with affine geometric stabilizers. Then there exist substacks $\mathfrak{F}_{1}, \ldots, \mathfrak{F}_{n}$ of $\mathfrak{F}$ with $\mathfrak{F}(\mathbb{K})=\coprod_{a=1}^{n} \mathfrak{F}_{a}(\mathbb{K})$, $\mathbb{K}$-varieties $Y_{1}, \ldots, Y_{n}$, and 1-morphisms $\phi_{a}: \mathfrak{F}_{a} \rightarrow Y_{a}$ with $\left(\phi_{a}\right)_{*}: \mathfrak{F}_{a}(\mathbb{K}) \rightarrow$ $Y_{a}(\mathbb{K})$ bijective for $a=1, \ldots, n$. 
Proof. By Theorem 2.7 $\mathfrak{F}$ can be stratified by global quotient stacks. Thus there exist finitely many substacks $U_{i}$ of $\mathfrak{F}$ with $\mathfrak{F}(\mathbb{K})=\coprod_{i} U_{i}(\mathbb{K})$, and each $U_{i}$ 1 -isomorphic to a quotient stack $\left[X_{i} / G_{i}\right]$, for $X_{i}$ a $\mathbb{K}$-variety and $G_{i}$ an affine algebraic $\mathbb{K}$-group acting on $X_{i}$. Theorem 2.3 gives a dense open $G_{i}$-invariant $X_{i}^{\prime} \subseteq X_{i}$, and a morphism of $\mathbb{K}$-varieties $\pi_{i}: X_{i}^{\prime} \rightarrow Y_{i}$ inducing a bijection $X_{i}^{\prime}(\mathbb{K}) / G_{i} \rightarrow Y_{i}(\mathbb{K})$. We may write $X_{i} \backslash X_{i}^{\prime}$ as a disjoint union of finitely many $G_{i}$-invariant $\mathbb{K}$-subvarieties $X_{i j}$ with $\operatorname{dim} X_{i j}<\operatorname{dim} X_{i}$. Applying Theorem 2.3 again gives a dense open $G_{i}$-invariant $X_{i j}^{\prime} \subseteq X_{i j}$ and a morphism of $\mathbb{K}$-varieties $\pi_{i j}: X_{i j}^{\prime} \rightarrow Y_{i j}$ inducing a bijection $X_{i j}^{\prime}(\mathbb{K}) / G_{i j} \rightarrow Y_{i j}(\mathbb{K})$.

As the dimension decreases at each stage, this process eventually yields finitely many substacks $\mathfrak{F}_{1}, \ldots, \mathfrak{F}_{n}$ of $\mathfrak{F}$ with $\mathfrak{F}(\mathbb{K})=\coprod_{a=1}^{n} \mathfrak{F}_{a}(\mathbb{K})$, such that $\mathfrak{F}_{a}$ is 1-isomorphic to $\left[X_{a} / G_{a}\right]$ for $\mathbb{K}$-varieties $X_{a}, Y_{a}$ and $G_{a}$ an algebraic $\mathbb{K}$ group acting on $X_{a}$, and morphisms $\pi_{a}: X_{a} \rightarrow Y_{a}$ inducing a bijection between $X_{a}(\mathbb{K}) / G_{a}$ and $Y_{a}(\mathbb{K})$. The 1 -isomorphisms $\mathfrak{F}_{a} \cong\left[X_{a} / G_{a}\right]$ and $\pi_{a}$ combine to give a 1-morphism $\phi_{a}: \mathfrak{F}_{a} \rightarrow Y_{a}$ with the properties we want.

We extend the last part of Proposition 3.3 to stacks.

Proposition 4.5. Let $\mathbb{K}$ be an algebraically closed field, $\mathfrak{F}, \mathfrak{G}$ be algebraic $\mathbb{K}$ stacks with affine geometric stabilizers, $\phi: \mathfrak{F} \rightarrow \mathfrak{G}$ be a 1-morphism, and $C \subseteq$ $\mathfrak{F}(\mathbb{K})$ be constructible. Then $\phi_{*}(C)$ is constructible in $\mathfrak{G}(\mathbb{K})$.

Proof. By Definition 4.1] $C=\bigcup_{i \in I} \mathfrak{F}_{i}(\mathbb{K})$, where $\left\{\mathfrak{F}_{i}: i \in I\right\}$ are finitely many finite type substacks $\mathfrak{F}_{i}$ of $\mathfrak{F}$. So by Lemma 4.2 it is enough to show each $\phi_{*}\left(\mathfrak{F}_{i}(\mathbb{K})\right)$ is constructible. As by convention $\mathfrak{G}$ is locally of finite type it admits an open cover $\left\{\mathfrak{G}_{j}: j \in J\right\}$ of finite type substacks $\mathfrak{G}_{j}$. By Proposition 4.4 for $a=1, \ldots, n_{j}$ there exist substacks $\mathfrak{G}_{j a}$ of $\mathfrak{G}_{j}, \mathbb{K}$-varieties $Y_{j a}$ and 1-morphisms $\psi_{j a}: \mathfrak{G}_{j a} \rightarrow Y_{j a}$ with $\left(\psi_{j a}\right)_{*}$ bijective, such that $\mathfrak{G}_{j}(\mathbb{K})=\coprod_{a=1}^{n_{j}} \mathfrak{G}_{j a}(\mathbb{K})$.

Now $\left\{\phi^{-1}\left(\mathfrak{G}_{j}\right): j \in J\right\}$ covers $\mathfrak{F}_{i}$, which is quasicompact as it is of finite type. So there exists a finite subset $J_{i} \subseteq J$ such that $\left\{\phi^{-1}\left(\mathfrak{G}_{j}\right): j \in J_{i}\right\}$ covers $\mathfrak{F}_{i}$. Set $\mathfrak{F}_{i j a}=\mathfrak{F}_{i} \cap \phi^{-1}\left(\mathfrak{G}_{j a}\right)$ for $j \in J_{i}$ and $a=1, \ldots, n_{j}$. Then $\mathfrak{F}_{i j a}$ is a finite type $\mathbb{K}$-substack, and $\phi_{i j a}=\left.\phi\right|_{\mathfrak{F}_{i j a}}: \mathfrak{F}_{i j a} \rightarrow \mathfrak{G}_{j a}$ a 1-morphism with $\phi_{*}\left(\mathfrak{F}_{i}(\mathbb{K})\right)=\bigcup_{j \in J_{i}} \bigcup_{a=1}^{n_{j}}\left(\phi_{i j a}\right)_{*}\left(\mathfrak{F}_{i j a}(\mathbb{K})\right)$. Hence by Lemma 4.2 it suffices to show $\left(\phi_{i j a}\right)_{*}\left(\mathfrak{F}_{i j a}(\mathbb{K})\right)$ is constructible in $\mathfrak{G}_{j a}(\mathbb{K})$ for all $i, j, a$.

As $\mathfrak{F}_{i j a}$ is finite type it has an atlas $u_{i j a}: U_{i j a} \rightarrow \mathfrak{F}_{i j a}$ with $U_{i j a}$ a finite type $\mathbb{K}$-scheme. Then $\psi_{j a} \circ \phi_{i j a} \circ u_{i j a}: U_{i j a} \rightarrow Y_{j a}$ is a morphism of $\mathbb{K}$-schemes. But $U_{i j a}(\mathbb{K})$ is constructible as $U_{i j a}$ is of finite type, so Proposition 3.3 shows

$$
\begin{aligned}
\left(\psi_{j a} \circ \phi_{i j a} \circ u_{i j a}\right)_{*}\left(U_{i j a}(\mathbb{K})\right) & =\left(\psi_{j a}\right)_{*} \circ\left(\phi_{i j a}\right)_{*} \circ\left(u_{i j a}\right)_{*}\left(U_{i j a}(\mathbb{K})\right) \\
& =\left(\psi_{j a}\right)_{*} \circ\left(\phi_{i j a}\right)_{*}\left(\mathfrak{F}_{i j a}(\mathbb{K})\right)
\end{aligned}
$$

is constructible in $Y_{j a}(\mathbb{K})$, where the second line follows since $\left(u_{i j a}\right)_{*}$ is surjective as $u_{i j a}$ is an atlas. Now $\psi_{j a}: \mathfrak{G}_{j a} \rightarrow Y_{j a}$ is a finite type 1-morphism, so it pulls back constructible subsets to constructible subsets. Therefore

$$
\left(\psi_{j a}\right)_{*}^{-1}\left(\left(\psi_{j a}\right)_{*} \circ\left(\phi_{i j a}\right)_{*}\left(\mathfrak{F}_{i j a}(\mathbb{K})\right)\right)=\left(\phi_{i j a}\right)_{*}\left(\mathfrak{F}_{i j a}(\mathbb{K})\right)
$$

is constructible in $\mathfrak{G}_{j a}(\mathbb{K})$, using $\left(\psi_{j a}\right)_{*}$ a bijection in the second step. 
Lemma 4.2 and Proposition 4.5 extend Proposition 3.3 to algebraic $\mathbb{K}$-stacks with affine geometric stabilizers. As the proof of Proposition 3.15 depended only on Proposition 3.3 it extends to such stacks.

Proposition 4.6. Let $\mathbb{K}$ be an algebraically closed field, and $\mathfrak{F}, \mathfrak{G}, \mathfrak{H}$ be algebraic $\mathbb{K}$-stacks with affine geometric stabilizers.

(a) Let $\phi: \mathfrak{F} \rightarrow \mathfrak{G}$ be a 1-morphism (1-isomorphism). Then $\phi_{*}: \mathfrak{F}(\mathbb{K}) \rightarrow$ $\mathfrak{G}(\mathbb{K})$ is a pseudomorphism (pseudoisomorphism).

(b) Let $S \subseteq \mathfrak{F}(\mathbb{K}), T \subseteq \mathfrak{G}(\mathbb{K})$ be locally constructible, $\Phi: S \rightarrow T$ be a pseudomorphism, and $C \subseteq S$ be constructible. Then $\Phi(C)$ is constructible in $\mathfrak{G}(\mathbb{K})$. Also, if $t \in T$ then $C \cap \Phi^{-1}(t)$ is constructible in $\mathfrak{F}(\mathbb{K})$. Hence, $\Phi^{-1}(t)$ is locally constructible in $\mathfrak{F}(\mathbb{K})$.

(c) Let $S \subseteq \mathfrak{F}(\mathbb{K}), T \subseteq \mathfrak{G}(\mathbb{K}), U \subseteq \mathfrak{H}(\mathbb{K})$ be locally constructible, and $\Phi$ : $S \rightarrow T, \Psi: T \rightarrow U$ be pseudo(iso)morphisms. Then $\Psi \circ \Phi: S \rightarrow U$ is a pseudo(iso)morphism.

The next proposition allows results about constructible sets and functions on schemes to be easily extended to stacks.

Proposition 4.7. Let $\mathbb{K}$ be an algebraically closed field, $\mathfrak{F}$ an algebraic $\mathbb{K}$-stack with affine geometric stabilizers, and $C \subseteq \mathfrak{F}(\mathbb{K})$ be constructible. Then $C$ is pseudoisomorphic to $Y(\mathbb{K})$ for a separated, finite type $\mathbb{K}$-scheme $Y$.

Proof. Write $C=\bigsqcup_{i \in I} \mathfrak{F}_{i}(\mathbb{K})$ for $\mathfrak{F}_{i}, i \in I$ finitely many finite type substacks in $\mathfrak{F}$. Proposition 4.4 gives substacks $\mathfrak{F}_{i a}$ in $\mathfrak{F}_{i}, \mathbb{K}$-varieties $Y_{i a}$ and 1-morphisms $\phi_{i a}: \mathfrak{F}_{i a} \rightarrow Y_{i a}$ for $a=1, \ldots, n_{i}$, with $\mathfrak{F}_{i}(\mathbb{K})=\coprod_{a=1}^{n_{i}} \mathfrak{F}_{i a}(\mathbb{K})$, and $\left(\phi_{i a}\right)_{*}$ bijective. Let $Y$ be the abstract disjoint union of the $Y_{i a}$ for $i \in I$ and $a=$ $1, \ldots, n_{i}$, as in Definition 2.2 It is a separated, finite type $\mathbb{K}$-scheme. Define $\Phi: C \rightarrow Y(\mathbb{K})$ by $\left.\Phi\right|_{\mathfrak{F}_{i a}(\mathbb{K})}=\left(\phi_{i a}\right)_{*}$ for all $i, a$. Then $\Phi$ is bijective, as $\left(\phi_{i a}\right)_{*}$ is. Proposition 4.6 (a) shows $\left(\phi_{i a}\right)_{*}$ is a pseudomorphism, so $\Phi$ is a pseudomorphism. As $\Phi$ is bijective and $C, Y(\mathbb{K})$ constructible, $\Phi$ is a pseudoisomorphism.

\subsection{The naïve Euler characteristic and pushforward}

Fix an algebraically closed field $\mathbb{K}$ of characteristic zero for the rest of the section. We consider the simplest generalization of $\chi(X), \chi(S, f), \mathrm{CF}(\Phi)$ to $\mathbb{K}$-stacks $\mathfrak{F}$, which we call naïve as it ignores the stabilizer groups $\operatorname{Iso}_{\mathbb{K}}(x)$ for $x \in \mathfrak{F}(\mathbb{K})$. Here is the analogue of Definitions 3.11 and 3.16

Definition 4.8. Let $\mathfrak{F}$ be an algebraic $\mathbb{K}$-stack with affine geometric stabilizers, and $C \subseteq \mathfrak{F}(\mathbb{K})$ be constructible. Then $C$ is pseudoisomorphic to $Y(\mathbb{K})$ for a separated, finite type $\mathbb{K}$-scheme $Y$ by Proposition 4.7 Define the naïve Euler characteristic $\chi^{\mathrm{na}}(C)$ by $\chi^{\mathrm{na}}(C)=\chi(Y)$, where $\chi(Y)$ is as in Definition 3.9 If $Y^{\prime}$ is another choice for $Y$ then $Y(\mathbb{K})$ is pseudoisomorphic to $Y^{\prime}(\mathbb{K})$ by Proposition 4.6] (c), so $\chi(Y)=\chi\left(Y^{\prime}\right)$. Thus $\chi^{\text {na }}(C)$ is well-defined. Now let 
$S \subseteq \mathfrak{F}(\mathbb{K})$ be locally constructible. For $f \in \mathrm{CF}(S)$, define the naïve weighted Euler characteristic $\chi^{\mathrm{na}}(S, f) \in \mathbb{Q}$ by

$$
\chi^{\mathrm{na}}(S, f)=\sum_{c \in f(S) \backslash\{0\}} c \chi^{\mathrm{na}}\left(f^{-1}(c)\right) .
$$

Suppose $\mathfrak{F}, \mathfrak{G}$ are algebraic $\mathbb{K}$-stacks with affine geometric stabilizers, $S \subseteq$ $\mathfrak{F}(\mathbb{K}), T \subseteq \mathfrak{G}(\mathbb{K})$ are locally constructible, $\Phi: S \rightarrow T$ is a pseudomorphism, and $f \in \mathrm{CF}(S)$. Define the naïve pushforward $\mathrm{CF}^{\text {na }}(\Phi) f: T \rightarrow \mathbb{Q}$ of $f$ to $T$ by

$$
\mathrm{CF}^{\mathrm{na}}(\Phi) f(t)=\chi^{\mathrm{na}}\left(S, f \cdot \delta_{\Phi^{-1}(t)}\right) \quad \text { for } t \in T .
$$

Here $\delta_{\Phi^{-1}(t)}$ is the characteristic function of $\Phi^{-1}(t) \subseteq S$ on $S$. As $\Phi^{-1}(t)$ is locally constructible by Proposition 4.6) (b) we have $\delta_{\Phi^{-1}(t)} \in \operatorname{LCF}(S)$, and $f \in \mathrm{CF}(S)$, so $f \cdot \delta_{\Phi^{-1}(t)} \in \mathrm{CF}(S)$. Thus (9) is well-defined.

Here are the naïve generalizations of Theorem 3.17 and Corollary 3.13

Theorem 4.9. Let $\mathfrak{F}, \mathfrak{G}, \mathfrak{H}$ be algebraic $\mathbb{K}$-stacks with affine geometric stabilizers, $S \subseteq \mathfrak{F}(\mathbb{K}), T \subseteq \mathfrak{G}(\mathbb{K}), U \subseteq \mathfrak{H}(\mathbb{K})$ be locally constructible, and $\Phi: S \rightarrow T$, $\Psi: T \rightarrow U$ be pseudomorphisms. If $f \in \mathrm{CF}(S)$, then $\mathrm{CF}^{\mathrm{na}}(\Phi) f$ is a constructible function on $T$. Thus $\mathrm{CF}^{\mathrm{na}}(\Phi): \mathrm{CF}(S) \rightarrow \mathrm{CF}(T)$ is a $\mathbb{Q}$-linear map. Also $\mathrm{CF}^{\mathrm{na}}(\Psi \circ \Phi)=\mathrm{CF}^{\mathrm{na}}(\Psi) \circ \mathrm{CF}^{\mathrm{na}}(\Phi)$ as linear maps $\mathrm{CF}(S) \rightarrow \mathrm{CF}(U)$.

Proof. Define $A=\operatorname{supp}(f) \subseteq S, B=\Phi(A) \subseteq T$ and $C=\Psi(B) \subseteq U$. Then $A$ is constructible by Definition 4.3 so $B, C$ are constructible by Proposition 4.6(b). By Proposition 4.7 there exist separated, finite type $\mathbb{K}$-schemes $X, Y, Z$ and pseudoisomorphisms $\alpha: A \rightarrow X(\mathbb{K}), \beta: B \rightarrow Y(\mathbb{K})$ and $\gamma: C \rightarrow Z(\mathbb{K})$. Then $f \circ \alpha^{-1} \in \mathrm{CF}(X)$ as $\left.f\right|_{A} \in \mathrm{CF}(A)$. By Proposition 4.6 (c), $\beta \circ \Phi \circ \alpha^{-1}: X(\mathbb{K}) \rightarrow$ $Y(\mathbb{K})$ and $\gamma \circ \Psi \circ \beta^{-1}: Y(\mathbb{K}) \rightarrow Z(\mathbb{K})$ are pseudomorphisms of $\mathbb{K}$-schemes, so Theorem 3.17 gives $\mathrm{CF}\left(\beta \circ \Phi \circ \alpha^{-1}\right)\left(f \circ \alpha^{-1}\right) \in \mathrm{CF}(Y)$ and

$$
\mathrm{CF}\left(\gamma \circ \Psi \circ \Phi \circ \alpha^{-1}\right)\left(f \circ \alpha^{-1}\right)=\mathrm{CF}\left(\gamma \circ \Psi \circ \beta^{-1}\right) \circ \mathrm{CF}\left(\beta \circ \Phi \circ \alpha^{-1}\right)\left(f \circ \alpha^{-1}\right) \text {. }
$$

Since $\beta, \gamma$ identify constructible sets and functions with constructible sets and functions, these easily imply $\left.\left(\mathrm{CF}^{\mathrm{na}}(\Phi) f\right)\right|_{B} \in \mathrm{CF}(B)$ and

$$
\left.\left(\mathrm{CF}^{\mathrm{na}}(\Psi \circ \Phi) f\right)\right|_{C}=\left.\left(\mathrm{CF}^{\mathrm{na}}(\Psi) \circ \mathrm{CF}^{\mathrm{na}}(\Phi) f\right)\right|_{C} .
$$

As the unrestricted functions are zero outside $B, C$, the theorem follows.

Corollary 4.10. Let $\mathfrak{F}, \mathfrak{G}$ be algebraic $\mathbb{K}$-stacks with affine geometric stabilizers, $S \subseteq \mathfrak{F}(\mathbb{K}), T \subseteq \mathfrak{G}(\mathbb{K})$ be locally constructible, $\Phi: S \rightarrow T$ be a pseudomorphism, and $f \in \mathrm{CF}(S)$. Then $\chi^{\mathrm{na}}(S, f)=\chi^{\mathrm{na}}\left(T, \mathrm{CF}^{\mathrm{na}}(\Phi) f\right)$.

\subsection{Stabilizers $\operatorname{IsO}_{\mathbb{K}}(x)$ and weight functions}

We now discuss how to modify the naïve Euler characteristic $\chi^{\text {na }}$ and pushforward $\mathrm{CF}^{\text {na }}$ of 4.3 to take account of stabilizer groups $\operatorname{Iso}_{\mathbb{K}}(x)$ for $x \in \mathfrak{F}(\mathbb{K})$. We do this by inserting a weight $w_{\mathfrak{F}}$ depending on $\operatorname{IsO}_{\mathbb{K}}(x)$. We continue to fix $\mathbb{K}$ algebraically closed of characteristic zero. 
Definition 4.11. Let $w$ : $\{$ affine algebraic $\mathbb{K}$-groups $G\} \rightarrow \mathbb{Q} \cup\{\infty\}, G \mapsto w(G)$ be a map with $w(G)=w\left(G^{\prime}\right)$ if $G \cong G^{\prime}$. If $\mathfrak{F}$ is an algebraic $\mathbb{K}$-stack with affine geometric stabilizers, define $w_{\mathfrak{F}}: \mathfrak{F}(\mathbb{K}) \rightarrow \mathbb{Q} \cup\{\infty\}$ by $w_{\mathfrak{F}}(x)=w\left(\operatorname{Iso}_{\mathbb{K}}(x)\right)$. We call $w$ an allowable weight function if $w_{\mathfrak{F}}$ is a locally constructible function on $\mathfrak{F}$ with values in $\mathbb{Q} \cup\{\infty\}$ for all $\mathfrak{F}$. We also call $w$ multiplicative if $w(G \times H)=$ $w(G) w(H)$ for all affine algebraic $\mathbb{K}$-groups $G, H$.

Here are the weighted analogues $\chi_{w}(C), \chi_{w}(S, f), \mathrm{CF}_{w}(\Phi) f$ of $\chi^{\mathrm{na}}(C)$, $\chi^{\mathrm{na}}(S, f), \mathrm{CF}^{\mathrm{na}}(\Phi) f$. We allow $w$ to take the values $0, \infty$ to accommodate the examples below. This means $\chi_{w}(C), \chi_{w}(S, f), \mathrm{CF}_{w}(\Phi) f$ are not always defined.

Definition 4.12. Let $w$ be an allowable weight function, $\mathfrak{F}, \mathfrak{G}$ algebraic $\mathbb{K}$ stacks with affine geometric stabilizers, $C \subseteq \mathfrak{F}(\mathbb{K})$ constructible, $S \subseteq \mathfrak{F}(\mathbb{K})$, $T \subseteq \mathfrak{G}(\mathbb{K})$ locally constructible, and $\Phi: S \rightarrow T$ a pseudomorphism.

If $w_{\mathfrak{F}} \neq \infty$ on $C$, define the $w$-Euler characteristic $\chi_{w}(C)=\chi^{\text {na }}\left(C,\left.w_{\mathfrak{F}}\right|_{C}\right)$. If $w_{\mathfrak{F}}(c)=\infty$ for some $c \in C$ we say $\chi_{w}(C)$ is undefined. For $f \in \operatorname{CF}(S)$ with $w_{\mathfrak{F}} \neq \infty$ on $\operatorname{supp} f$, define the weighted $w$-Euler characteristic $\chi_{w}(S, f)$ by $\chi_{w}(S, f)=\chi^{\text {na }}\left(S, w_{\mathfrak{F}} f\right)=\chi^{\text {na }}\left(\operatorname{supp} f, w_{\mathfrak{F}} f\right)$, taking $w_{\mathfrak{F}} f=0$ outside $\operatorname{supp} f$ even where $w_{\mathfrak{F}}=\infty$. If $w_{\mathfrak{F}}(s)=\infty$ for some $s \in \operatorname{supp} f$ we say $\chi_{w}(S, f)$ is undefined. If $w_{\mathfrak{F}} \neq \infty$ on $S$ and $w_{\mathfrak{G}} \neq 0$ on $T$ then $w_{\mathfrak{F}} \in \operatorname{LCF}(S)$ and $w_{\mathfrak{G}}^{-1} \in \operatorname{LCF}(T)$ by Definition 4.11 Define

$$
\mathrm{CF}_{w}(\Phi) f=w_{\mathfrak{G}}^{-1} \cdot \mathrm{CF}^{\mathrm{na}}(\Phi)\left(w_{\mathfrak{F}} f\right) \quad \text { for } f \in \mathrm{CF}(S) .
$$

This is well-defined in $\mathrm{CF}(T)$ as $w_{\mathfrak{F}} f \in \mathrm{CF}(S)$, so $\mathrm{CF}^{\text {na }}(\Phi)\left(w_{\mathfrak{F}} f\right) \in \operatorname{CF}(T)$. Therefore $\mathrm{CF}_{w}(\Phi): \mathrm{CF}(S) \rightarrow \mathrm{CF}(T)$ is a $\mathbb{Q}$-linear map. If $w_{\mathfrak{F}}(s)=\infty$ for some $s \in S$ or $w_{\mathfrak{G}}(t)=0$ for some $t \in T$, we say $\mathrm{CF}_{w}(\Phi)$ is undefined.

Then $\chi_{w}$ satisfies the following analogues of Theorem 3.10(ii),(iii):

Lemma 4.13. Let $w$ be an allowable weight function, and $\mathfrak{F}, \mathfrak{G}$ algebraic $\mathbb{K}$ stacks with affine geometric stabilizers. Then

(i) Suppose $C, D_{1}, \ldots, D_{m} \subseteq \mathfrak{F}(\mathbb{K})$ are constructible with $C=\coprod_{i=1}^{m} D_{i}$. Then $\chi_{w}(C)=\sum_{i=1}^{m} \chi_{w}\left(D_{i}\right)$ if either side is defined.

(ii) If $w$ is multiplicative and $C \subseteq \mathfrak{F}(\mathbb{K}), D \subseteq \mathfrak{G}(\mathbb{K})$ are constructible then $\chi_{w}(C \times D)=\chi_{w}(C) \chi_{w}(D)$ if both sides are defined.

For the analogue of Theorem 4.9 from (10) we have

$$
\begin{aligned}
\mathrm{CF}_{w}(\Psi \circ \Phi) f & =w_{\mathfrak{H}}^{-1} \cdot \mathrm{CF}^{\mathrm{na}}(\Psi \circ \Phi)\left(w_{\mathfrak{F}} f\right)=w_{\mathfrak{H}}^{-1} \cdot \mathrm{CF}^{\mathrm{na}}(\Psi) \circ \mathrm{CF}^{\mathrm{na}}(\Phi)\left(w_{\mathfrak{F}} f\right) \\
& =w_{\mathfrak{H}}^{-1} \cdot \mathrm{CF}^{\mathrm{na}}(\Psi)\left[w_{\mathfrak{G}} \cdot \mathrm{CF}_{w}(\Phi) f\right]=\mathrm{CF}_{w}(\Psi) \circ \mathrm{CF}_{w}(\Phi) f
\end{aligned}
$$

by Theorem 4.9 provided everything is defined. So we deduce:

Corollary 4.14. Let $w$ be an allowable weight function, $\mathfrak{F}, \mathfrak{G}, \mathfrak{H}$ algebraic $\mathbb{K}$ stacks with affine geometric stabilizers, $S \subseteq \mathfrak{F}(\mathbb{K}), T \subseteq \mathfrak{G}(\mathbb{K}), U \subseteq \mathfrak{H}(\mathbb{K})$ locally constructible with $w_{\mathfrak{F}} \neq \infty$ on $S, w_{\mathfrak{G}} \neq 0, \infty$ on $T, w_{\mathfrak{H}} \neq 0$ on $U$, and $\Phi: S \rightarrow T$, $\Psi: T \rightarrow U$ be pseudomorphisms. Then $\mathrm{CF}_{w}(\Psi \circ \Phi)=\mathrm{CF}_{w}(\Psi) \circ \mathrm{CF}_{w}(\Phi)$. 
As in Corollary 4.10 we have:

Corollary 4.15. Let $w$ be an allowable weight function, $\mathfrak{F}, \mathfrak{G}$ algebraic $\mathbb{K}$-stacks with affine geometric stabilizers, $S \subseteq \mathfrak{F}(\mathbb{K}), T \subseteq \mathfrak{G}(\mathbb{K})$ locally constructible with $w_{\mathfrak{F}} \neq \infty$ on $S, w_{\mathfrak{G}} \neq 0, \infty$ on $T, \Phi: S \rightarrow T$ a pseudomorphism, and $f \in \mathrm{CF}(S)$. Then $\chi_{w}(S, f)=\chi_{w}\left(T, \mathrm{CF}_{w}(\Phi) f\right)$.

Here are two examples of multiplicative allowable weight functions.

Proposition 4.16. (a) Define e : $\{$ affine algebraic $\mathbb{K}$-groups $\} \rightarrow \mathbb{Z}$ by $e(G)=$ $\chi(G)$. Then $e$ is a multiplicative allowable weight function.

(b) If $G$ is an affine algebraic $\mathbb{K}$-group, define the adjoint action of $G$ on itself by $\operatorname{Ad}(g) h=g h g^{-1}$. Then the quotient $[G / \operatorname{Ad}(G)]$ is an algebraic $\mathbb{K}$-stack of finite type. Define $o(G)=\chi^{\mathrm{na}}([G / \operatorname{Ad}(G)])$. Then o : \{affine algebraic $\mathbb{K}$ groups $\} \rightarrow \mathbb{Z}$ is a multiplicative allowable weight function.

Proof. Clearly $e$ and $o$ are well-defined and multiplicative. Let $\mathfrak{F}$ be an algebraic $\mathbb{K}$-stack with affine geometric stabilizers. We must show $e_{\mathfrak{F}}, o_{\mathfrak{F}} \in \operatorname{LCF}(\mathfrak{F})$, which holds provided $e_{\mathfrak{G}}=\left.e_{\mathfrak{F}}\right|_{\mathfrak{G}(\mathbb{K})}$ and $o_{\mathfrak{G}}=\left.o_{\mathfrak{F}}\right|_{\mathfrak{G}(\mathbb{K})}$ lie in $\mathrm{CF}(\mathfrak{G})$ for all finite type $\mathbb{K}$ substacks $\mathfrak{G}$ in $\mathfrak{F}$. Theorem[2.7 gives $\mathfrak{G}(\mathbb{K})=\coprod_{i \in I} U_{i}(\mathbb{K})$, where $\left\{U_{i}: i \in I\right\}$ are finitely many substacks of $\mathfrak{G}$ with $U_{i} 1$-isomorphic to $\left[X_{i} / G_{i}\right]$ for $X_{i}$ a $\mathbb{K}$-variety and $G_{i}$ an affine algebraic $\mathbb{K}$-group, acting on $X_{i}$ by $\rho_{i}: X_{i} \times G_{i} \rightarrow X_{i}$.

Write $\pi_{i}: X_{i} \rightarrow U_{i}$ for the projection 1-morphism. Let $Y_{i}$ be the inverse image under $\operatorname{id}_{X_{i}} \times \rho_{i}: X_{i} \times G_{i} \rightarrow X_{i} \times X_{i}$ of the diagonal in $X_{i} \times X_{i}$. Then $Y_{i}$ is a finite type closed subscheme of $X_{i} \times G_{i}$. Let $\sigma_{i}: Y_{i} \rightarrow X_{i}$ be the restriction of the projection $X_{i} \times G_{i} \rightarrow X_{i}$. Then for each $x \in X_{i}(\mathbb{K}), \sigma_{i}^{-1}(x)=\{x\} \times \operatorname{Stab}_{x}\left(G_{i}\right)$, where $\operatorname{Stab}_{x}\left(G_{i}\right)$ is the stabilizer subgroup of $x$ in $G_{i}$.

Theorem 3.17 gives $\mathrm{CF}\left(\sigma_{i}\right) 1 \in \mathrm{CF}\left(X_{i}\right)$, as $1 \in \mathrm{CF}\left(Y_{i}\right)$. But for $x \in X_{i}(\mathbb{K})$

$$
\left(\mathrm{CF}\left(\sigma_{i}\right) 1\right)(x)=\chi\left[\operatorname{Stab}_{x}\left(G_{i}\right)\right]=\chi\left[\operatorname{Iso}_{\mathbb{K}}\left(\left(\pi_{i}\right)_{*}(x)\right)\right]=e_{\mathfrak{G}}\left(\left(\pi_{i}\right)_{*}(x)\right),
$$

as $\sigma_{i}^{-1}(x)=\{x\} \times \operatorname{Stab}_{x}\left(G_{i}\right)$, and the stabilizer group $\operatorname{IsOK}_{\mathbb{K}}\left(\left(\pi_{i}\right)_{*}(x)\right)$ in $U_{i} \cong$ $\left[X_{i} / G_{i}\right]$ is $\operatorname{Stab}_{x}\left(G_{i}\right)$. Therefore $\mathrm{CF}\left(\pi_{i}\right) 1=e_{\mathfrak{G}} \circ\left(\pi_{i}\right)_{*}$ as maps $X_{i}(\mathbb{K}) \rightarrow \mathbb{Q}$. Since $\left(\pi_{i}\right)_{*}$ is surjective this implies that $\left.e_{\mathfrak{G}}\right|_{U_{i}(\mathbb{K})} \in \mathrm{CF}\left(U_{i}\right)$, as $\left(\pi_{i}\right)_{*}$ takes constructible sets to constructible sets by Proposition 4.5 But $\mathfrak{G}(\mathbb{K})=\bigsqcup_{i \in I} U_{i}(\mathbb{K})$ and $I$ is finite, so $e_{\mathfrak{G}} \in \mathrm{CF}(\mathfrak{G})$. This proves (a).

For (b), we form an algebraic $\mathbb{K}$-stack $\mathfrak{H}_{i}$ with 1-morphisms $Y_{i} \stackrel{\alpha_{i}}{\longrightarrow} \mathfrak{H}_{i} \stackrel{\beta_{i}}{\longrightarrow} X_{i}$ with $\pi_{i}=\beta_{i} \circ \alpha_{i}$, such that if $x \in X_{i}(\mathbb{K})$ with $\operatorname{Stab}_{x}\left(G_{i}\right)=H$, so that $\pi_{i}^{-1}(x)=$ $\{x\} \times H$, then $\beta_{i}^{-1}(x)=\{x\} \times[H / \operatorname{Ad}(H)]$, and $\alpha_{i}: \pi_{i}^{-1}(x) \rightarrow \beta_{i}^{-1}(x)$ is the projection $H \rightarrow[H / \operatorname{Ad}(H)]$. Then $\alpha_{i}$ is an atlas, so $\mathfrak{H}_{i}$ is of finite type. Thus $1 \in \mathrm{CF}\left(\mathfrak{H}_{i}\right)$, so $\mathrm{CF}^{\text {na }}\left(\beta_{i}\right) 1 \in \mathrm{CF}\left(X_{i}\right)$ by Theorem 4.9 But for $x \in X_{i}(\mathbb{K})$

$$
\begin{aligned}
\left(\operatorname{CF}^{\mathrm{na}}\left(\beta_{i}\right) 1\right)(x) & =\chi^{\mathrm{na}}\left(\left[\operatorname{Stab}_{x}\left(G_{i}\right) / \operatorname{Ad}\left(\operatorname{Stab}_{x}\left(G_{i}\right)\right)\right]\right) \\
& =\chi^{\mathrm{na}}\left(\left[\operatorname{Iso}_{\mathbb{K}}\left(\left(\pi_{i}\right)_{*}(x)\right) / \operatorname{Ad}\left(\operatorname{Iso}_{\mathbb{K}}\left(\left(\pi_{i}\right)_{*}(x)\right)\right)\right]\right)=o_{\mathfrak{G}}\left(\left(\pi_{i}\right)_{*}(x)\right) .
\end{aligned}
$$

The rest of the proof is as for (a).

Other weight functions constructed from $e, o$ in a multiplicative way are also multiplicative and allowable, such as $e^{k}, o^{k},|e|^{k},|o|^{k}, \operatorname{sign}(e), \operatorname{sign}(o)$ and $e^{k} o^{l}$ for $k, l \in \mathbb{Z}$ with $k l>0$. We give special names to two interesting cases. 
Definition 4.17. Let $w$ be an allowable weight function, $\mathfrak{F}, \mathfrak{G}$ algebraic $\mathbb{K}$-stacks with affine geometric stabilizers, $C \subseteq \mathfrak{F}(\mathbb{K})$ constructible, $S \subseteq \mathfrak{F}(\mathbb{K}), T \subseteq \mathfrak{G}(\mathbb{K})$ locally constructible, $\Phi: S \rightarrow T$ a pseudomorphism, and $f \in \mathrm{CF}(S)$.

(a) Define the stack (weighted) Euler characteristic by $\chi^{\text {stk }}(C)=\chi_{1 / e}(C)$ and $\chi^{\text {stk }}(S, f)=\chi_{1 / e}(S, f)$, and the stack pushforward $\mathrm{CF}^{\text {stk }}(\Phi)=\mathrm{CF}_{1 / e}(\Phi)$, in the sense of Definition 4.11 where $e$ is the weight function of Proposition 4.16(a), and $1 / e$ is allowable. Here $\chi^{\text {stk }}(C)$ is only defined if $\chi\left(\operatorname{Iso}_{\mathbb{K}}(c)\right) \neq 0$ for all $c \in C$, and $\chi^{\text {stk }}(S, f)$ only if $\chi\left(\operatorname{Iso}_{\mathbb{K}}(s)\right) \neq 0$ for all $s \in \operatorname{supp} f$, and $\mathrm{CF}^{\text {stk }}(\Phi)$ only if $\chi\left(\operatorname{Iso}_{\mathbb{K}}(s)\right) \neq 0$ for all $s \in S$.

(b) Define the orbifold (weighted) Euler characteristic by $\chi^{\mathrm{orb}}(C)=\chi_{o}(C)$ and $\chi^{\mathrm{orb}}(S, f)=\chi_{o}(S, f)$, and the orbifold pushforward $\mathrm{CF}^{\mathrm{orb}}(\Phi)=$ $\mathrm{CF}_{o}(\Phi)$, where $o$ is the allowable weight function of Proposition 4.16(b). As $o$ takes values in $\mathbb{Z}, \chi^{\text {orb }}(C)$ and $\chi^{\text {orb }}(S, f)$ are always defined, and $\mathrm{CF}^{\mathrm{orb}}(\Phi)$ is defined if $o\left(\operatorname{Iso}_{\mathbb{K}}(t)\right) \neq 0$ for all $t \in T$.

The stack Euler characteristic $\chi^{\text {stk }}$ and its pushforward $\mathrm{CF}^{\text {stk }}$ turn out to be the natural notions for the problems in [9-11]. If $X$ is a $\mathbb{K}$-variety and $G$ an algebraic $\mathbb{K}$-group acting on $X$ with $\chi(G) \neq 0$, then $\chi^{\text {stk }}([X / G])=\chi(X) / \chi(G)$. It also has a universal property in Cartesian squares, in $\$ 5.2$

Unfortunately, as $\chi(G)=0$ for any algebraic $\mathbb{K}$-group $G$ with $\mathbb{K}^{\times}=\mathbb{K} \backslash\{0\}$ as a subgroup, $\chi^{\text {stk }}(C), \chi^{\text {stk }}(S, f)$ and $\mathrm{CF}^{\text {stk }}(\Phi)$ above are undefined in many interesting situations, including everything in [9-11]. But in $\$ 5.1$ we will extend the definition of $\mathrm{CF}^{\text {stk }}(\Phi)$ to $\mathrm{CF}^{\text {stk }}(\phi)$ for $\phi: \mathfrak{F} \rightarrow \mathfrak{G}$ a representable 1 -morphism, and this will be sufficient for the applications of [9-11].

For Deligne-Mumford stacks all stabilizer groups are finite, and for $G$ finite $\chi(G)=|G|>0$, so that $\chi^{\text {stk }}, \mathrm{CF}^{\text {stk }}$ are always defined. It is well-established that for enumerative problems on Deligne-Mumford stacks one counts a point $x \in \mathfrak{F}(\mathbb{K})$ with weight $1 /\left|\operatorname{Iso}_{\mathbb{K}}(x)\right|$, and $\chi^{\text {stk }}$ generalizes this approach.

The orbifold Euler characteristic is the author's attempt to generalize to stacks something already well understood for orbifolds. Let $G$ be a finite group acting on a compact manifold $M$, so that $M / G$ is an orbifold. Dixon et al. [2, p. 684] observe the correct Euler characteristic of $M / G$ in String Theory is

$$
\chi(M, G)=\frac{1}{|G|} \sum_{g, h \in G: g h=h g} \chi\left(M^{g, h}\right),
$$

where $M^{g, h}=\{x \in M: g \cdot x=h \cdot x=x\}$.

Atiyah and Segal [1] later interpreted $\chi(M, G)$ as the Euler characteristic of equivariant $K$-theory $K_{G}(M)$. For a survey and further references on orbifold Euler characteristics, see Roan [20]. In particular, it is believed and in many cases known that for a complex orbifold $M / G, \chi(M, G)$ coincides with the Euler characteristic $\chi(X)$ of any crepant resolution of $M / G$.

Let $M$ be a $\mathbb{K}$-scheme acted on by a finite group $G$. Then $M^{g, h}$ is a subscheme of $M$, and (11) makes sense. An easy calculation shows $\chi(M, G)=$ 
$\chi^{\mathrm{na}}([M / G], f)$, where $f \in \mathrm{CF}([M / G])$ is given by

$$
\begin{aligned}
f(x) & =\frac{\left|\left\{(g, h) \in \operatorname{Iso}_{\mathbb{K}}(x)^{2}: g h=h g\right\}\right|}{\left|\operatorname{Iso}_{\mathbb{K}}(x)\right|}=\sum_{g \in \operatorname{Iso}_{\mathbb{K}}(x)} \frac{\left|\left\{h \in \operatorname{Iso}_{\mathbb{K}}(x): g h=h g\right\}\right|}{\left|\operatorname{Iso}_{\mathbb{K}}(x)\right|} \\
& =\sum_{g \in \operatorname{Iso}_{\mathbb{K}}(x)} \frac{1}{\left|\operatorname{Ad}\left(\operatorname{Iso}_{\mathbb{K}}(x)\right) g\right|}=\left|\operatorname{Iso}_{\mathbb{K}}(x) / \operatorname{Ad}\left(\operatorname{Iso}_{\mathbb{K}}(x)\right)\right|=o_{[M / G]}(x)
\end{aligned}
$$

for $x \in[M / G](\mathbb{K})$. Hence $\chi(M, G)=\chi^{\text {orb }}([M / G])$ by Definitions 4.11] and 4.17]

Thus, our orbifold Euler characteristic $\chi^{\text {orb }}([M / G])$ of the Deligne-Mumford stack $[M / G]$ agrees with the physicists' orbifold Euler characteristic $\chi(M, G)$ of the complex orbifold $M / G$ when $\mathbb{K}=\mathbb{C}$, but our notion $\chi^{\text {orb }}$ is also defined over other fields $\mathbb{K}$ and for more general stacks $\mathfrak{F}$. It would be interesting to know whether $\chi(M, G)$ being the Euler characteristic of any crepant resolution over $\mathbb{C}$ extends using $\chi^{\text {orb }}$ to other fields, or to more general stacks.

\section{Representable and finite type 1-morphisms}

Next we study stack pushforwards $\mathrm{CF}^{\text {stk }}(\phi)$ by 1-morphisms $\phi: \mathfrak{F} \rightarrow \mathfrak{G}$. Then $\phi_{*}: \mathfrak{F}(\mathbb{K}) \rightarrow \mathfrak{G}(\mathbb{K})$ is a pseudomorphism, so the obvious definition is $\mathrm{CF}^{\text {stk }}(\phi)=$ $\mathrm{CF}^{\mathrm{stk}}\left(\phi_{*}\right)$. However, $\mathrm{CF}^{\mathrm{stk}}\left(\phi_{*}\right)$ is undefined if $x \in \mathfrak{F}(\mathbb{K})$ with $\chi\left(\operatorname{Iso}_{\mathbb{K}}(x)\right)=0$. Since $\chi(G)=0$ for many affine algebraic $\mathbb{K}$-groups $G$, this is a serious drawback. Instead, by using the extra data of the homomorphisms $\phi_{*}: \operatorname{IsO}_{\mathbb{K}}(x) \rightarrow$ $\operatorname{Iso}_{\mathbb{K}}\left(\phi_{*}(x)\right)$, in 5.1 we define $\mathrm{CF}^{\text {stk }}(\phi)$ in many cases when $\mathrm{CF}^{\text {stk }}\left(\phi_{*}\right)$ is undefined, in particular for all representable $\phi$.

Section 5.2 defines the pullback $\psi^{*}: \mathrm{CF}(\mathfrak{G}) \rightarrow \mathrm{CF}(\mathfrak{F})$ for a finite type 1morphism $\psi: \mathfrak{F} \rightarrow \mathfrak{G}$, and proves pullbacks $\psi^{*}$ and pushforwards $\mathrm{CF}^{\text {stk }}(\phi)$ commute in Cartesian squares. This will be an important tool in [9-11]. In $\$ 5.3$. for finite type $\phi: \mathfrak{F} \rightarrow \mathfrak{G}$ we extend $\mathrm{CF}^{\text {na }}\left(\phi_{*}\right), \mathrm{CF}^{\text {stk }}(\phi)$ to locally constructible functions, with the usual functorial property.

Fix an algebraically closed field $\mathbb{K}$ of characteristic zero for all of this section.

\subsection{Pushforwards by representable 1-morphisms}

Here is our definition of the stack pushforward $\mathrm{CF}^{\text {stk }}(\phi)$ for a 1-morphism $\phi$.

Definition 5.1. Let $\mathfrak{F}, \mathfrak{G}$ be algebraic $\mathbb{K}$-stacks with affine geometric stabilizers and $\phi: \mathfrak{F} \rightarrow \mathfrak{G}$ a 1-morphism. Then for any $x \in \mathfrak{F}(\mathbb{K})$ we have a morphism $\phi_{*}: \operatorname{IsO}_{\mathbb{K}}(x) \rightarrow \operatorname{Iso}_{\mathbb{K}}\left(\phi_{*}(x)\right)$ of affine algebraic $\mathbb{K}$-groups. The kernel $\operatorname{Ker} \phi_{*}$ is an affine algebraic $\mathbb{K}$-group in $\operatorname{IsO}_{\mathbb{K}}(x)$, so $\chi\left(\operatorname{Ker} \phi_{*}\right)$ is defined. The image $\phi_{*}\left(\operatorname{Iso}_{\mathbb{K}}(x)\right)$ is an affine algebraic $\mathbb{K}$-group closed in $\operatorname{Iso}_{\mathbb{K}}\left(\phi_{*}(x)\right)$, so the quotient $\operatorname{IsO}_{\mathbb{K}}\left(\phi_{*}(x)\right) / \phi_{*}\left(\operatorname{IsO}_{\mathbb{K}}(x)\right)$ is a quasiprojective $\mathbb{K}$-variety. Thus $\chi\left(\operatorname{Iso}_{\mathbb{K}}\left(\phi_{*}(x)\right) / \phi_{*}\left(\operatorname{IsO}_{\mathbb{K}}(x)\right)\right)$ is also defined.

Suppose $\chi\left(\operatorname{Ker} \phi_{*}\right) \neq 0$ for all $x \in \mathfrak{F}(\mathbb{K})$. Define $m_{\phi}: \mathfrak{F}(\mathbb{K}) \rightarrow \mathbb{Q}$ by

$$
m_{\phi}(x)=\frac{\chi\left(\operatorname{Iso}_{\mathbb{K}}\left(\phi_{*}(x)\right) / \phi_{*}\left(\operatorname{Iso}_{\mathbb{K}}(x)\right)\right)}{\chi\left(\operatorname{Ker}\left(\phi_{*}: \operatorname{Iso}_{\mathbb{K}}(x) \rightarrow \operatorname{Iso}_{\mathbb{K}}\left(\phi_{*}(x)\right)\right)\right)} \quad \text { for } x \in \mathfrak{F}(\mathbb{K}) .
$$


An argument similar to Proposition 4.16] shows $m_{\phi} \in \operatorname{LCF}(\mathfrak{F})$. Define the stack pushforward $\mathrm{CF}^{\text {stk }}(\phi): \mathrm{CF}(\mathfrak{F}) \rightarrow \mathrm{CF}(\mathfrak{G})$ by

$$
\mathrm{CF}^{\mathrm{stk}}(\phi) f=\mathrm{CF}^{\mathrm{na}}\left(\phi_{*}\right)\left(m_{\phi} \cdot f\right) .
$$

Here $m_{\phi} \cdot f \in \mathrm{CF}(\mathfrak{F})$ as $m_{\phi} \in \operatorname{LCF}(\mathfrak{F})$ and $f \in \mathrm{CF}(\mathfrak{F})$, so (13) is well-defined, and $\mathrm{CF}^{\text {stk }}(\phi): \mathrm{CF}(\mathfrak{F}) \rightarrow \mathrm{CF}(\mathfrak{G})$ is $\mathbb{Q}$-linear.

This agrees with the previous definition of $\mathrm{CF}^{\text {stk }}\left(\phi_{*}\right)$ when it is defined, regarding $\phi_{*}: \mathfrak{F}(\mathbb{K}) \rightarrow \mathfrak{G}(\mathbb{K})$ as a pseudomorphism by Proposition 4.6(a).

Lemma 5.2. In Definition 5.1] if $\mathrm{CF}^{\mathrm{stk}}\left(\phi_{*}\right)$ is defined in Definition [.17 then $\mathrm{CF}^{\text {stk }}(\phi): \mathrm{CF}(\mathfrak{F}) \rightarrow \mathrm{CF}(\mathfrak{G})$ is defined and $\mathrm{CF}^{\text {stk }}\left(\phi_{*}\right)=\mathrm{CF}^{\text {stk }}(\phi)$.

Proof. Suppose $\mathrm{CF}^{\text {stk }}\left(\phi_{*}\right)$ is defined. Then $1 / e_{\mathfrak{F}} \neq \infty$ in $\mathfrak{F}(\mathbb{K})$, so $\chi\left(\operatorname{Iso}_{\mathbb{K}}(x)\right) \neq 0$ for $x \in \mathfrak{F}(\mathbb{K})$. Now $\operatorname{Ker} \phi_{*}$ is normal in $\operatorname{Iso}_{\mathbb{K}}(x)$, with quotient $\operatorname{Iso}_{\mathbb{K}}(x) / \operatorname{Ker} \phi_{*}$ naturally isomorphic to $\phi_{*}\left(\operatorname{Iso}_{\mathbb{K}}(x)\right)$. Hence

$$
\chi\left(\operatorname{Iso}_{\mathbb{K}}(x)\right)=\chi\left(\operatorname{Ker} \phi_{*}\right) \cdot \chi\left(\phi_{*}\left(\operatorname{Iso}_{\mathbb{K}}(x)\right)\right),
$$

by general properties of $\chi$. As $\chi\left(\operatorname{IsOK}_{\mathbb{K}}(x)\right) \neq 0$ this implies $\chi\left(\operatorname{Ker} \phi_{*}\right) \neq 0$ for $x \in \mathfrak{F}(\mathbb{K})$, so $\mathrm{CF}^{\text {stk }}(\phi): \mathrm{CF}(\mathfrak{F}) \rightarrow \mathrm{CF}(\mathfrak{G})$ is defined. Similarly, we have

$$
\chi\left(\operatorname{IsO}_{\mathbb{K}}\left(\phi_{*}(x)\right)\right)=\chi\left(\phi_{*}\left(\operatorname{IsO}_{\mathbb{K}}(x)\right)\right) \cdot \chi\left(\operatorname{IsO}_{\mathbb{K}}\left(\phi_{*}(x)\right) / \phi_{*}\left(\operatorname{Iso}_{\mathbb{K}}(x)\right)\right) .
$$

Dividing this equation by the previous one for $x \in \mathfrak{F}(\mathbb{K})$, which is valid as $\chi(\operatorname{IsOKK}(x)) \neq 0$, and using (12) gives

$$
m_{\phi}(x)=\chi\left(\operatorname{Iso}_{\mathbb{K}}\left(\phi_{*}(x)\right) / \chi\left(\operatorname{Iso}_{\mathbb{K}}(x)\right)=e_{\mathfrak{G}}\left(\phi_{*}(x)\right) / e_{\mathfrak{F}}(x) \quad \text { for } x \in \mathfrak{F}(\mathbb{K}) .\right.
$$

It follows immediately from Definition 4.17 that $\mathrm{CF}^{\text {stk }}\left(\phi_{*}\right)=\mathrm{CF}^{\text {stk }}(\phi)$.

The functorial behaviour of Theorem 4.9 holds for $\mathrm{CF}^{\text {stk }}(\phi)$.

Theorem 5.3. Let $\mathfrak{F}, \mathfrak{G}, \mathfrak{H}$ be algebraic $\mathbb{K}$-stacks with affine geometric stabilizers, and $\phi: \mathfrak{F} \rightarrow \mathfrak{G}, \psi: \mathfrak{G} \rightarrow \mathfrak{H} 1$-morphisms. Suppose the kernels of $\phi_{*}$ : $\operatorname{IsO}_{\mathbb{K}}(x) \rightarrow \operatorname{IsO}_{\mathbb{K}}\left(\phi_{*}(x)\right)$ for $x \in \mathfrak{F}(\mathbb{K})$ and $\psi_{*}: \operatorname{IsO}_{\mathbb{K}}(y) \rightarrow \operatorname{IsO}_{\mathbb{K}}\left(\psi_{*}(y)\right)$ for $y \in \mathfrak{G}(\mathbb{K})$ have nonzero Euler characteristics. Then $\mathrm{CF}^{\mathrm{stk}}(\psi \circ \phi)=\mathrm{CF}^{\text {stk }}(\psi) \circ \mathrm{CF}^{\text {stk }}(\phi)$ as well-defined linear maps $\mathrm{CF}(\mathfrak{F}) \rightarrow \mathrm{CF}(\mathfrak{H})$.

Proof. Let $x \in \mathfrak{F}(\mathbb{K})$, and set $y=\phi_{*}(x)$ and $z=\psi_{*}(y)$. Write

$$
\begin{gathered}
G_{x}=\operatorname{IsO}_{\mathbb{K}}(x), \quad G_{y}=\operatorname{IsO}_{\mathbb{K}}(y), \quad G_{z}=\operatorname{Iso}_{\mathbb{K}}(z), \quad \phi_{x}=\phi_{*}: G_{x} \rightarrow G_{y}, \\
\psi_{y}=\psi_{*}: G_{y} \rightarrow G_{z}, \quad K_{\phi, x}=\operatorname{Ker}\left(\phi_{x}\right), \quad K_{\psi, y}=\operatorname{Ker}\left(\psi_{y}\right), \quad K_{\psi \circ \phi, x}=\operatorname{Ker}\left(\psi_{y} \circ \phi_{x}\right), \\
I_{\phi, x}=\operatorname{Image}\left(\phi_{x}\right), \quad I_{\psi, y}=\operatorname{Image}\left(\psi_{y}\right), \quad I_{\psi \circ \phi, x}=\operatorname{Image}\left(\psi_{y} \circ \phi_{x}\right) .
\end{gathered}
$$

Then $K_{\phi, x}$ is normal in $K_{\psi \circ \phi, x}$, and the quotient $K_{\psi \circ \phi, x} / K_{\phi, x}$ is isomorphic to $I_{\phi, x} \cap K_{\psi, y}$. So general properties of $\chi$ give

$$
\chi\left(K_{\psi \circ \phi, x}\right)=\chi\left(K_{\phi, x}\right) \cdot \chi\left(I_{\phi, x} \cap K_{\psi, y}\right) .
$$


The inclusions $I_{\phi, x} \cap K_{\psi, y} \subseteq K_{\psi, y}$ and $I_{\psi \circ \phi, x} \subseteq I_{\psi, y} \subseteq G_{z}$ imply that

$$
\begin{aligned}
\chi\left(K_{\psi, y}\right) & =\chi\left(K_{\psi, y} /\left(I_{\phi, x} \cap K_{\psi, y}\right)\right) \cdot \chi\left(I_{\phi, x} \cap K_{\psi, y}\right) \\
\text { and } \quad \chi\left(G_{z} / I_{\psi \circ \phi, x}\right) & =\chi\left(G_{z} / I_{\psi, y}\right) \cdot \chi\left(I_{\psi, y} / I_{\psi \circ \phi, x}\right) .
\end{aligned}
$$

By assumption $\chi\left(K_{\phi, x}\right), \chi\left(K_{\psi, y}\right) \neq 0$ for $x \in \mathfrak{F}(\mathbb{K})$ and $y \in \mathfrak{G}(\mathbb{K})$, so $\mathrm{CF}^{\text {stk }}(\phi): \mathrm{CF}(\mathfrak{F}) \rightarrow \mathrm{CF}(\mathfrak{G})$ and $\mathrm{CF}^{\text {stk }}(\psi): \mathrm{CF}(\mathfrak{G}) \rightarrow \mathrm{CF}(\mathfrak{H})$ are defined. As $\chi\left(K_{\psi, y}\right) \neq 0$ equation (15) gives $\chi\left(I_{\phi, x} \cap K_{\psi, y}\right) \neq 0$, and this, $\chi\left(K_{\phi, x}\right) \neq 0$ and (14) show that $\chi\left(K_{\psi \circ \phi, x}\right) \neq 0$, which holds for all $x \in \mathfrak{F}(\mathbb{K})$. Hence $\mathrm{CF}^{\text {stk }}(\psi \circ \phi): \mathrm{CF}(\mathfrak{F}) \rightarrow \mathrm{CF}(\mathfrak{H})$ is defined.

Now $\psi_{x}^{-1}\left(I_{\psi \circ \phi, x}\right)$ is an algebraic group with $I_{\phi, x} \subseteq \psi_{x}^{-1}\left(I_{\psi \circ \phi, x}\right) \subseteq G_{y}$, so

$$
\chi\left(G_{y} / I_{\phi, x}\right)=\chi\left(G_{y} / \psi_{x}^{-1}\left(I_{\psi \circ \phi, x}\right)\right) \cdot \chi\left(\psi_{x}^{-1}\left(I_{\psi \circ \phi, x}\right) / I_{\phi, x}\right) .
$$

But $\psi_{x}$ and $\gamma\left(I_{\phi, x} \cap K_{\psi, y}\right) \mapsto \gamma I_{\phi, x}$ induce isomorphisms of homogeneous spaces

$$
G_{y} / \psi_{x}^{-1}\left(I_{\psi \circ \phi, x}\right) \cong I_{\psi, y} / I_{\psi \circ \phi, x}, \quad K_{\psi, y} /\left(I_{\phi, x} \cap K_{\psi, y}\right) \cong \psi_{x}^{-1}\left(I_{\psi \circ \phi, x}\right) / I_{\phi, x} .
$$

Therefore the last two equations give

$$
\chi\left(G_{y} / I_{\phi, x}\right)=\chi\left(I_{\psi, y} / I_{\psi \circ \phi, x}\right) \cdot \chi\left(K_{\psi, y} /\left(I_{\phi, x} \cap K_{\psi, y}\right)\right) .
$$

Combining equations (12) and (14)-(17) yields

$$
\begin{aligned}
m_{\psi \circ \phi}(x) & =\frac{\chi\left(G_{z} / I_{\psi \circ \phi, x}\right)}{\chi\left(K_{\psi \circ \phi, x}\right)}=\frac{\chi\left(G_{z} / I_{\psi, y}\right) \chi\left(I_{\psi, y} / I_{\psi \circ \phi, x}\right)}{\chi\left(K_{\phi, x}\right) \chi\left(I_{\phi, x} \cap K_{\psi, y}\right)} \cdot \frac{\chi\left(K_{\psi, y} /\left(I_{\phi, x} \cap K_{\psi, y}\right)\right)}{\chi\left(K_{\psi, y} /\left(I_{\phi, x} \cap K_{\psi, y}\right)\right)} \\
& =\frac{\chi\left(G_{z} / I_{\psi, y}\right)}{\chi\left(K_{\psi, y}\right)} \cdot \frac{\chi\left(G_{y} / I_{\phi, x}\right)}{\chi\left(K_{\phi, x}\right)}=m_{\psi}(y) \cdot m_{\phi}(x) .
\end{aligned}
$$

This identity is easily seen to be the extra ingredient needed to modify the proof of Theorem 4.9 to prove that $\mathrm{CF}^{\text {stk }}(\psi \circ \phi)=\mathrm{CF}^{\text {stk }}(\psi) \circ \mathrm{CF}^{\text {stk }}(\phi)$.

If $\phi: \mathfrak{F} \rightarrow \mathfrak{G}$ is representable then $\phi_{*}: \operatorname{Iso}_{\mathbb{K}}(x) \rightarrow \operatorname{Iso}_{\mathbb{K}}\left(\phi_{*}(x)\right)$ is an injective morphism of algebraic $\mathbb{K}$-groups for all $x \in \mathfrak{F}(\mathbb{K})$. Thus $\operatorname{Ker} \phi_{*}=\{1\}$, so $\chi\left(\operatorname{Ker} \phi_{*}\right)=1 \neq 0$ for all $x \in \mathfrak{F}(\mathbb{K})$, and $\mathrm{CF}^{\text {stk }}(\phi)$ is defined. This gives:

Theorem 5.4. Let $\mathfrak{F}, \mathfrak{G}, \mathfrak{H}$ be algebraic $\mathbb{K}$-stacks with affine geometric stabilizers, and $\phi: \mathfrak{F} \rightarrow \mathfrak{G}, \psi: \mathfrak{G} \rightarrow \mathfrak{H}$ representable 1-morphisms. Then $\psi \circ \phi: \mathfrak{F} \rightarrow \mathfrak{H}$ is representable, and $\mathrm{CF}^{\text {stk }}(\phi): \mathrm{CF}(\mathfrak{F}) \rightarrow \mathrm{CF}(\mathfrak{G}), \mathrm{CF}^{\text {stk }}(\psi):$ $\mathrm{CF}(\mathfrak{G}) \rightarrow \mathrm{CF}(\mathfrak{H})$ and $\mathrm{CF}^{\text {stk }}(\psi \circ \phi): \mathrm{CF}(\mathfrak{F}) \rightarrow \mathrm{CF}(\mathfrak{H})$ are well-defined linear maps with $\mathrm{CF}^{\text {stk }}(\psi \circ \phi)=\mathrm{CF}^{\text {stk }}(\psi) \circ \mathrm{CF}^{\text {stk }}(\phi)$.

Also, for $\phi$ representable $m_{\phi}$ in (12) takes values in $\mathbb{Z}$, so $\mathrm{CF}^{\text {stk }}(\phi)$ maps $\mathbb{Z}$-valued functions $\mathrm{CF}(\mathfrak{F})_{\mathbb{Z}} \subset \mathrm{CF}(\mathfrak{F})$ to $\mathbb{Z}$-valued functions $\mathrm{CF}(\mathfrak{G})_{\mathbb{Z}} \subset \mathrm{CF}(\mathfrak{G})$. 


\subsection{Pullbacks by finite type 1-morphisms}

For finite type $\phi: \mathfrak{F} \rightarrow \mathfrak{G}$ we can pull back constructible functions from $\mathfrak{G}$ to $\mathfrak{F}$.

Definition 5.5. Suppose $\phi: \mathfrak{F} \rightarrow \mathfrak{G}$ is a finite type 1 -morphism of algebraic $\mathbb{K}$ stacks and $C \subseteq \mathfrak{G}(\mathbb{K})$ is constructible. Then $C=\bigcup_{i \in I} \mathfrak{G}_{i}(\mathbb{K})$, where $\left\{\mathfrak{G}_{i}: i \in I\right\}$ are finitely many substacks of $\mathfrak{G}$. Set $\mathfrak{F}_{i}=\phi^{*}\left(\mathfrak{G}_{i}\right)$, a $\mathbb{K}$-substack of $\mathfrak{F}$. Then $\mathfrak{F}_{i}$ is of finite type, as $\phi, \mathfrak{G}_{i}$ are. Hence $\phi_{*}^{-1}(C)=\bigcup_{i \in I} \phi_{*}^{-1}\left(\mathfrak{G}_{i}(\mathbb{K})\right)=\bigcup_{i \in I} \mathfrak{F}_{i}(\mathbb{K})$ is constructible in $\mathfrak{F}$. That is, $\phi_{*}: \mathfrak{F}(\mathbb{K}) \rightarrow \mathfrak{G}(\mathbb{K})$ pulls back constructible sets to constructible sets. Thus if $f \in \mathrm{CF}(\mathfrak{G})$ then $f \circ \phi_{*}$ lies in $\mathrm{CF}(\mathfrak{F})$. Define the pullback $\phi^{*}: \mathrm{CF}(\mathfrak{G}) \rightarrow \mathrm{CF}(\mathfrak{F})$ by $\phi^{*}(f)=f \circ \phi_{*}$. Pullbacks commute with multiplication of functions, that is, $\phi^{*}(f g)=\phi^{*}(f) \phi^{*}(g)$. If also $\psi: \mathfrak{G} \rightarrow \mathfrak{H}$ is a finite type 1-morphism, it is immediate that $(\psi \circ \phi)^{*}=\phi^{*} \circ \psi^{*}: \mathrm{CF}(\mathfrak{H}) \rightarrow \mathrm{CF}(\mathfrak{F})$.

It is an interesting question how pullbacks $\psi^{*}$ and pushforwards $\mathrm{CF}^{\mathrm{stk}}(\phi)$ are related. The next theorem shows they commute in Cartesian squares, as in Definition 2.5 It will be an important tool in [9-11]. The theorem would not hold if we replaced $\mathrm{CF}^{\text {stk }}(\eta), \mathrm{CF}^{\text {stk }}(\phi)$ in (18) by $\mathrm{CF}^{\text {na }}(\eta), \mathrm{CF}^{\text {na }}(\phi)$, or pushforwards defined using some other weight function. This supports our claim that $\mathrm{CF}^{\text {stk }}$ is the most natural pushforward in many stack problems.

Theorem 5.6. Let $\mathfrak{E}, \mathfrak{F}, \mathfrak{G}, \mathfrak{H}$ be algebraic $\mathbb{K}$-stacks with affine geometric stabilizers. If

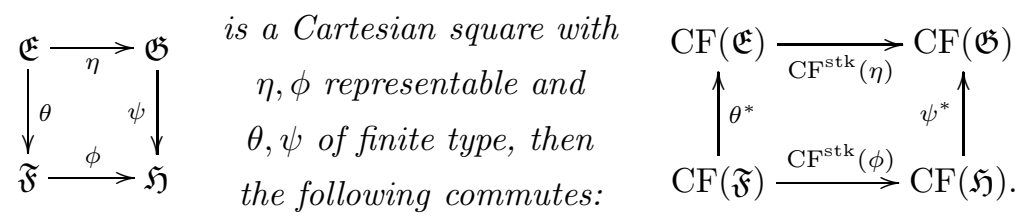

Proof. Let $C \subseteq \mathfrak{F}(\mathbb{K})$ be constructible, and $\delta_{C} \in \mathrm{CF}(\mathfrak{F})$ be its characteristic function. We shall prove that

$$
\left(\mathrm{CF}^{\mathrm{stk}}(\eta) \circ \theta^{*}\right) \delta_{C}=\left(\psi^{*} \circ \mathrm{CF}^{\mathrm{stk}}(\phi)\right) \delta_{C} .
$$

As $\mathrm{CF}^{\text {stk }}(\eta) \circ \theta^{*}, \psi^{*} \circ \mathrm{CF}^{\mathrm{stk}}(\phi)$ are linear and such $\delta_{C}$ generate $\mathrm{CF}(\mathfrak{F})$, this implies $\mathrm{CF}^{\mathrm{stk}}(\eta) \circ \theta^{*}=\psi^{*} \circ \mathrm{CF}^{\text {stk }}(\phi)$, as we want.

Define $B=\theta_{*}^{-1}(C)$. Since $\theta$ is of finite type $B$ is constructible, as in Definition [5.5] and $\theta^{*}\left(\delta_{C}\right)=\delta_{B}$, the characteristic function of $B$. Let $x \in \mathfrak{G}(\mathbb{K})$, and define $y=\psi_{*}(x), B_{x}=B \cap \eta_{*}^{-1}(\{x\})$ and $C_{y}=C \cap \phi_{*}^{-1}(\{y\})$. Then $B_{x}, C_{y}$ are constructible, as $B, C$ are and $\eta_{*}^{-1}(\{x\}), \phi_{*}^{-1}(\{y\})$ are locally constructible. Write $\delta_{B_{x}}, \delta_{C_{y}}, \delta_{\eta_{*}^{-1}(x)}, \delta_{\phi_{*}^{-1}(y)}$ for the characteristic functions. Then

$$
\begin{gathered}
\left(\left(\mathrm{CF}^{\mathrm{stk}}(\eta) \circ \theta^{*}\right) \delta_{C}\right)(x)=\left(\mathrm{CF}^{\mathrm{stk}}(\eta)\left(\delta_{C} \circ \theta_{*}\right)\right)(x)=\left(\mathrm{CF}^{\mathrm{stk}}(\eta) \delta_{B}\right)(x)= \\
\left(\mathrm{CF}^{\mathrm{na}}\left(\eta_{*}\right)\left(m_{\eta} \cdot \delta_{B}\right)\right)(x)=\chi^{\mathrm{na}}\left(\mathfrak{E}, m_{\eta} \cdot \delta_{B_{x}}\right)=\chi^{\mathrm{na}}\left(\mathfrak{F}, \mathrm{CF}^{\mathrm{na}}(\theta)\left(m_{\eta} \cdot \delta_{B_{x}}\right)\right),
\end{gathered}
$$

by (9), (13) and Corollary 4.10, where $m_{\eta}$ is defined in (12). Similarly we have

$$
\left(\left(\psi^{*} \circ \mathrm{CF}^{\mathrm{stk}}(\phi)\right) \delta_{C}\right)(x)=\chi^{\mathrm{na}}\left(\mathfrak{F}, m_{\phi} \cdot \delta_{C_{y}}\right) .
$$


We shall prove that

$$
\mathrm{CF}^{\mathrm{na}}(\theta)\left(m_{\eta} \cdot \delta_{B_{x}}\right)=m_{\phi} \cdot \delta_{C_{y}} \quad \text { in } \mathrm{CF}(\mathfrak{F}) .
$$

If $z \in \mathfrak{F}(\mathbb{K}) \backslash C_{y}$ then both sides of (22) are zero at $z$. So let $z \in C_{y}$. Then $\theta_{*}^{-1}(\{z\}) \cap B_{x}=\theta_{*}^{-1}(\{z\}) \cap \eta_{*}^{-1}(\{x\})$, so by (9) equation (22) at $z$ reduces to

$$
\chi^{\mathrm{na}}\left(\mathfrak{E}, m_{\eta} \cdot \delta_{\eta_{*}^{-1}(x)} \cdot \delta_{\theta_{*}^{-1}(z)}\right)=m_{\phi}(z) .
$$

Define $G_{x}=\operatorname{Iso}_{\mathbb{K}}(x), G_{y}=\operatorname{IsO}_{\mathbb{K}}(y)$ and $G_{z}=\operatorname{IsO}_{\mathbb{K}}(z)$, as algebraic $\mathbb{K}$-groups. Since $\psi_{*}(x)=y$ and $\phi_{*}(z)=y$ we have homomorphisms $\psi_{*}: \operatorname{Iso}_{\mathbb{K}}(x) \rightarrow \operatorname{IsO}_{\mathbb{K}}(y)$

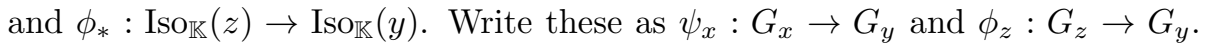
Then $\phi_{z}$ is injective, as $\phi$ is representable, so $\chi\left(\operatorname{Ker} \phi_{z}\right)=\{1\}$ and (12) gives

$$
m_{\phi}(z)=\chi\left(G_{y} / \phi_{z}\left(G_{z}\right)\right) .
$$

As (18) is Cartesian $\mathfrak{E}$ is 1 -isomorphic to $\mathfrak{F} \times_{\mathfrak{H}} \mathfrak{G}$ by Definition 2.5 By definition of fibre products we find $\eta_{*}^{-1}(\{x\}) \cap \theta_{*}^{-1}(\{z\})$ is naturally isomorphic to $\psi_{x}\left(G_{x}\right) \backslash G_{y} / \phi_{z}\left(G_{z}\right)$, a biquotient. The stabilizer groups are given by

$$
\operatorname{IsO}_{\mathbb{K}}\left(\psi_{x}\left(G_{x}\right) \beta \phi_{z}\left(G_{z}\right)\right)=\left\{(\alpha, \gamma) \in G_{x} \times G_{y}: \psi_{x}(\alpha) \beta=\beta \phi_{z}(\gamma)\right\} \quad \text { for } \beta \in G_{y} \text {, }
$$

and the group homomorphism $\eta_{*}: \operatorname{Iso}_{\mathbb{K}}\left(\psi_{x}\left(G_{x}\right) \beta \phi_{z}\left(G_{z}\right)\right) \rightarrow \operatorname{Iso}_{\mathbb{K}}(x)=G_{x}$ is given by $(\alpha, \gamma) \mapsto \alpha$. It is injective as $\phi_{z}$ is injective. Thus (12) yields

$$
m_{\eta}\left(\psi_{x}\left(G_{x}\right) \beta \phi_{z}\left(G_{z}\right)\right)=\chi\left(G_{x} /\left\{\alpha \in G_{x}: \psi_{x}(\alpha) \beta \phi_{z}\left(G_{z}\right)=\beta \phi_{z}\left(G_{z}\right)\right\}\right) .
$$

Let $\Pi_{x, y, z}: G_{y} / \phi_{z}\left(G_{z}\right) \rightarrow \psi_{x}\left(G_{x}\right) \backslash G_{y} / \phi_{z}\left(G_{z}\right)$ be the natural projection. Then the fibre of $\Pi_{x, y, z}$ over $\psi_{x}\left(G_{x}\right) \beta \phi_{z}\left(G_{z}\right)$ is isomorphic to $G_{x} /\left\{\alpha \in G_{x}\right.$ : $\left.\psi_{x}(\alpha) \beta \phi_{z}\left(G_{z}\right)=\beta \phi_{z}\left(G_{z}\right)\right\}$. So (25) implies that $\mathrm{CF}^{\mathrm{na}}\left(\Pi_{x, y, z}\right) 1=m_{\eta}$ in $\mathrm{CF}\left(\psi_{x}\left(G_{x}\right) \backslash G_{y} / \phi_{z}\left(G_{z}\right)\right)$. Therefore

$$
\begin{gathered}
\chi^{\mathrm{na}}\left(\mathfrak{E}, m_{\eta} \cdot \delta_{\eta_{*}^{-1}(x)} \cdot \delta_{\theta_{*}^{-1}(z)}\right)=\chi^{\mathrm{na}}\left(\psi_{x}\left(G_{x}\right) \backslash G_{y} / \phi_{z}\left(G_{z}\right), m_{\eta}\right)= \\
\chi^{\mathrm{na}}\left(\psi_{x}\left(G_{x}\right) \backslash G_{y} / \phi_{z}\left(G_{z}\right), \mathrm{CF}^{\mathrm{na}}\left(\Pi_{x, y, z}\right) 1\right)=\chi^{\mathrm{na}}\left(G_{y} / \phi_{z}\left(G_{z}\right), 1\right)=m_{\phi}(z),
\end{gathered}
$$

by Corollary 4.10 and (24). This proves (23), and hence (22). Equations (20)(22) then give (19) at $x$, as we have to prove.

Note that by [15, Rem. 4.14.1 \& Lem. $3.11 \&$ Rem. 4.17(2)], in a Cartesian square (18) of algebraic $\mathbb{K}$-stacks, if $\phi$ is representable then $\eta$ is representable, and if $\psi$ is of finite type then $\theta$ is of finite type. Thus it is enough to suppose only that $\phi$ is representable and $\psi$ of finite type in (18).

\subsection{Pushforwards of locally constructible functions}

Next we observe that if $\phi: \mathfrak{F} \rightarrow \mathfrak{G}$ is of finite type then the definitions of $\mathrm{CF}^{\mathrm{na}}\left(\phi_{*}\right) f, \mathrm{CF}^{\mathrm{stk}}(\phi) f$ in (9), (13) make sense for $f$ only locally constructible. 
Definition 5.7. Let $\phi: \mathfrak{F} \rightarrow \mathfrak{G}$ be a finite type 1-morphism of algebraic $\mathbb{K}$-stacks with affine geometric stabilizers. For $f \in \operatorname{LCF}(\mathfrak{F})$, define $\operatorname{LCF}^{\text {na }}(\phi) f$ by

$$
\operatorname{LCF}^{\mathrm{na}}(\phi) f(x)=\chi^{\mathrm{na}}\left(\mathfrak{F}, f \cdot \delta_{\phi_{*}^{-1}(x)}\right) \quad \text { for } x \in \mathfrak{F}(\mathbb{K}),
$$

following (9). This is well-defined as $\phi_{*}^{-1}(\{x\})$ is constructible since $\phi$ is of finite type. Thus $\delta_{\phi_{*}^{-1}(x)} \in \mathrm{CF}(\mathfrak{F})$ and $f \in \operatorname{LCF}(\mathfrak{F})$, giving $f \cdot \delta_{\phi_{*}^{-1}(x)} \in \mathrm{CF}(\mathfrak{F})$. If $\phi$ is also representable, define $\operatorname{LCF}^{\mathrm{stk}}(\phi) f=\mathrm{LCF}^{\mathrm{na}}(\phi)\left(m_{\phi} \cdot f\right)$ as in (13).

Suppose $C \subseteq \mathfrak{G}(\mathbb{K})$ is constructible, and let $B=\phi_{*}^{-1}(C)$. Then $B$ is constructible as $\phi$ is of finite type, by Definition 5.5 Write $\delta_{B}, \delta_{C}$ for the characteristic functions of $B, C$. Then $f \cdot \delta_{B} \in \mathrm{CF}(\mathfrak{F})$, and it follows easily that

$$
\left(\operatorname{LCF}^{\mathrm{na}}(\phi) f\right) \delta_{C}=\mathrm{CF}^{\mathrm{na}}\left(\phi_{*}\right)\left(f \cdot \delta_{B}\right) \text { and }\left(\mathrm{LCF}^{\mathrm{stk}}(\phi) f\right) \delta_{C}=\mathrm{CF}^{\mathrm{stk}}(\phi)\left(f \cdot \delta_{B}\right) \text {. }
$$

Therefore $\left.\left(\operatorname{LCF}^{\text {na }}(\phi) f\right)\right|_{C},\left.\left(\operatorname{LCF}^{\text {stk }}(\phi) f\right)\right|_{C}$ are constructible by Theorems 4.9 and 5.4 for any constructible $C \subseteq \mathfrak{G}(\mathbb{K})$. Hence $\operatorname{LCF}^{\text {na }}(\phi) f, \operatorname{LCF}^{\text {stk }}(\phi) f$ are locally constructible, and $\operatorname{LCF}^{\mathrm{na}}(\phi), \mathrm{LCF}^{\text {stk }}(\phi)$ are linear maps $\operatorname{LCF}(\mathfrak{F}) \rightarrow$ $\operatorname{LCF}(\mathfrak{G})$. From Theorems 4.9 and 5.4 we deduce:

Theorem 5.8. Let $\mathfrak{F}, \mathfrak{G}, \mathfrak{H}$ be algebraic $\mathbb{K}$-stacks with affine geometric stabilizers, and $\phi: \mathfrak{F} \rightarrow \mathfrak{G}, \psi: \mathfrak{G} \rightarrow \mathfrak{H}$ finite type 1-morphisms. Then so is $\psi \circ \phi$, and $\mathrm{LCF}^{\mathrm{na}}(\psi \circ \phi)=\mathrm{LCF}^{\mathrm{na}}(\psi) \circ \mathrm{LCF}^{\mathrm{na}}(\phi)$. If $\phi, \psi$ are representable then so is $\psi \circ \phi$, and $\mathrm{LCF}^{\text {stk }}(\psi \circ \phi)=\mathrm{LCF}^{\text {stk }}(\psi) \circ \mathrm{LCF}^{\text {stk }}(\phi)$.

The locally constructible analogue of Theorem [5.6] also holds.

\section{References}

[1] M. Atiyah and G. Segal, On equivariant Euler characteristics, J. Geom. Phys. 6 (1989), 671-677.

[2] L. Dixon, J.A. Harvey, C. Vafa and E. Witten, Strings on orbifolds, Nucl. Phys. B261 (1985), 678-686.

[3] T.L. Gómez, Algebraic stacks, Proc. Indian Acad. Sci. Math. Sci. 111 (2001), 1-31. math.AG/9911199.

[4] A. Grothendieck and others, Séminaire de Géométrie Algébrique. Part 4: Théorie des topos et cohomologie étale des schémas, Lecture Notes in Math. 269, 270 and 305, Springer, Heidelberg, 1972-3. Part 4 $\frac{1}{2}$ : Cohomologie étale (by P. Deligne), Lecture Notes in Math. 569, Springer, Heidelberg, 1977. Part 5: Cohomologie l-adique et fonctions L, Lecture Notes in Math. 589, Springer, Heidelberg, 1977.

[5] R. Hartshorne, Algebraic Geometry, Graduate Texts in Math. 52, SpringerVerlag, New York, 1977. 
[6] L. Illusie, Théorie de Brauer et caractéristique d'Euler-Poincaré d'après P. Deligne, Exp. VIII in Astérisque 82-83, 1981.

[7] D.D. Joyce, Motivic invariants of Artin stacks and 'stack functions', math.AG/0509722 version 2, 2006.

[8] D.D. Joyce, Configurations in abelian categories. I. Basic properties and moduli stacks, math.AG/0312190, version 5, 2005.

[9] D.D. Joyce, Configurations in abelian categories. II. Ringel-Hall algebras, math.AG/0503029 version 3, 2006.

[10] D.D. Joyce, Configurations in abelian categories. III. Stability conditions and identities, math.AG/0410267, version 4, 2006.

[11] D.D. Joyce, Configurations in abelian categories. IV. Invariants and changing stability conditions, math.AG/0410268, version 4, 2006.

[12] N.M. Katz and G. Laumon, Transformation de Fourier et majoration des sommes exponentielles, Publ. Math. I.H.E.S. 62 (1985), 145-202.

[13] G. Kennedy, MacPherson's Chern classes of singular algebraic varieties, Communications in Algebra 18 (1990), 2821-2839.

[14] A. Kresch, Cycle groups for Artin stacks, Invent. math. 138 (1999), 495536. math.AG/9810166

[15] G. Laumon and L. Moret-Bailly, Champs algébriques, Ergeb. der Math. und ihrer Grenzgebiete 39, Springer-Verlag, Berlin, 2000.

[16] R.D. MacPherson, Chern classes for singular algebraic varieties, Ann. Math. 100 (1974), 423-432.

[17] D. Marker, Model Theory: An Introduction, Graduate Texts in Math. 217, Springer, New York, 2002.

[18] J.S. Milne, Étale Cohomology, Princeton University Press, Princeton, 1980.

[19] D. Mumford, The Red Book of Varieties and Schemes, second edition, Lecture Notes in Math. 1358, Springer-Verlag, Berlin, 1999.

[20] S.-S. Roan, Orbifold Euler Characteristic. In B. Greene and S.-T. Yau, editors, Mirror Symmetry II, pages 129-140. A.M.S. and International Press, 1997.

[21] M. Rosenlicht, A Remark on Quotient Spaces, Annaes da Academia Brasileira de Ciêncas 35 (1963), 487-489.

[22] O.Y. Viro, Some integral calculus based on Euler characteristic. In O.Y. Viro, editor, Topology and Geometry - Rohlin seminar, Lecture Notes in Math. 1346, pages 127-138. Springer-Verlag, Berlin, 1988. 